\title{
Cancellous bone and theropod dinosaur locomotion. Part II - A new approach to inferring posture and locomotor biomechanics in extinct tetrapod vertebrates
}

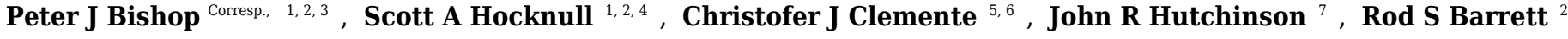 \\ 3, David G Lloyd ${ }^{2,3}$ \\ ${ }^{1}$ Geosciences Program, Queensland Museum, Brisbane, Queensland, Australia \\ 2 School of Allied Health Sciences, Griffith University, Gold Coast, Queensland, Australia \\ ${ }^{3}$ Gold Coast Orthopaedic Research, Engineering and Education Alliance, Menzies Health Institute Queensland, Australia \\ 4 School of Biosciences, University of Melbourne, Melbourne, Victoria, Australia \\ 5 School of Science and Engineering, University of the Sunshine Coast, Maroochydore, Queensland, Australia \\ 6 School of Biological Sciences, University of Queensland, Brisbane, Queensland, Australia \\ 7 Structure and Motion Laboratory, Department of Comparative Biomedical Sciences, Royal Veterinary College, Hatfield, Hertfordshire, United Kingdom \\ Corresponding Author: Peter J Bishop \\ Email address: peter.bishop@qm.qld.gov.au
}

This paper is the second of a three-part series that investigates the architecture of cancellous bone in the main hindlimb bones of theropod dinosaurs, and uses cancellous bone architectural patterns to infer locomotor biomechanics in extinct non-avian species. Cancellous bone is widely known to be highly sensitive to its mechanical environment, and therefore has the potential to provide insight into locomotor biomechanics in extinct tetrapod vertebrates such as dinosaurs. Here in Part II, a new biomechanical modelling approach is outlined, one which mechanistically links cancellous bone architectural patterns with three-dimensional musculoskeletal and finite element modelling of the hindlimb. In particular, the architecture of cancellous bone is used to derive a single 'characteristic posture' for a given species - one in which bone continuum-level principal stresses best align with cancellous bone fabric - and thereby clarify hindlimb locomotor biomechanics. The quasi-static approach was validated for an extant theropod, the chicken, and is shown to provide a good estimate of limb posture at around mid-stance. It also provides reasonable predictions of bone loading mechanics, especially for the proximal hindlimb, and also provides a broadly accurate assessment of muscle recruitment insofar as limb stabilization is concerned. In addition to being useful for better understanding locomotor biomechanics in extant species, the approach hence provides a new avenue by which to analyse, test and refine palaeobiomechanical hypotheses, not just for extinct theropods, but potentially many other extinct tetrapod groups as well. 


\section{Cancellous bone and theropod dinosaur locomotion.}

2 Part II - A new approach to inferring posture and

3 locomotor biomechanics in extinct tetrapod

4 vertebrates

5

6

P.J. Bishop ${ }^{1,2,3, *}$, S.A. Hocknull ${ }^{1,2,4}$, C.J. Clemente ${ }^{5,6}$, J.R. Hutchinson ${ }^{7}$, R.S. Barrett ${ }^{2,3}$ and D.G. Lloyd $^{2,3}$.

${ }^{1}$ Geosciences Program, Queensland Museum, Brisbane, Queensland, Australia.

${ }^{2}$ School of Allied Health Sciences, Griffith University, Gold Coast, Queensland, Australia.

${ }^{3}$ Gold Coast Orthopaedic Research, Engineering and Education Alliance, Menzies Health Institute Queensland.

${ }^{4}$ School of Biosciences, University of Melbourne, Melbourne, Victoria, Australia.

${ }^{5}$ School of Science and Engineering, University of the Sunshine Coast, Maroochydore, Queensland, Australia.

${ }^{6}$ School of Biological Sciences, The University of Queensland, Brisbane, Queensland, Australia. ${ }^{7}$ Structure and Motion Laboratory, Department of Comparative Biomedical Sciences, Royal Veterinary College, Hatfield, Hertfordshire, UK.

*corresponding author at current address: Structure and Motion Laboratory, Department of Comparative Biomedical Sciences, Royal Veterinary College, Hatfield, Hertfordshire, UK; pbishop@rvc.ac.uk. 


\section{II.1 Abstract}

31

This paper is the second of a three-part series that investigates the architecture of cancellous bone in the main hindlimb bones of theropod dinosaurs, and uses cancellous bone architectural patterns to infer locomotor biomechanics in extinct non-avian species. Cancellous bone is widely known to be highly sensitive to its mechanical environment, and therefore has the potential to provide insight into locomotor biomechanics in extinct tetrapod vertebrates such as dinosaurs. Here in Part II, a new biomechanical modelling approach is outlined, one which mechanistically links cancellous bone architectural patterns with three-dimensional musculoskeletal and finite element modelling of the hindlimb. In particular, the architecture of cancellous bone is used to derive a single 'characteristic posture' for a given species - one in which bone continuum-level principal stresses best align with cancellous bone fabric - and thereby clarify hindlimb locomotor biomechanics. The quasi-static approach was validated for an extant theropod, the chicken, and is shown to provide a good estimate of limb posture at around mid-stance. It also provides reasonable predictions of bone loading mechanics, especially for the proximal hindlimb, and also provides a broadly accurate assessment of muscle recruitment insofar as limb stabilization is concerned. In addition to being useful for better understanding locomotor biomechanics in extant species, the approach hence provides a new avenue by which to analyse, test and refine palaeobiomechanical hypotheses, not just for extinct theropods, but potentially many other extinct tetrapod groups as well. 


\section{II.2 Introduction}

63

Cancellous bone is highly sensitive and able to adapt its three-dimensional (3-D) architecture to its prevailing mechanical environment, such that the overall architecture strongly reflects the loads experienced by whole bones. Cancellous bone architecture can also change when loading conditions change, and that the structural alteration takes place in a predictable fashion (Adachi et al. 2001; Barak et al. 2011; Biewener et al. 1996; Goldstein et al. 1991; Guldman et al. 1997; Huiskes et al. 2000; Mullender \& Huiskes 1995; Polk et al. 2008; Pontzer et al. 2006; Radin et al. 1982; Richmond et al. 2005; Ruimerman et al. 2005; van der Meulen et al. 2006; van der Meulen et al. 2009; Volpato et al. 2008; Wang et al. 2012). Furthermore, comparative studies have shown that differences in loading conditions, resulting from differences in locomotor behaviour and biomechanics, are often reflected as differences in architectural patterns between species (Amson et al. 2017; Barak et al. 2013; Fajardo \& Müller 2001; Griffin et al. 2010; Hébert et al. 2012; MacLatchy \& Müller 2002; Maga et al. 2006; Matarazzo 2015; Ryan \& Ketcham 2002; Ryan \& Ketcham 2005; Ryan \& Shaw 2012; Su et al. 2013; Tsegai et al. 2013). Locomotor-dependent architectural differences have also been borne out in Part I of this series, which has highlighted a number of important differences in cancellous bone architecture between the hindlimb bones of humans and birds, the two kinds of obligate, striding bipeds alive today (Bishop et al. in reviewb).

As outlined in Part I of this series, the overarching paradigm that relates cancellous bone architectural fabric to its mechanical environment is the 'trajectorial theory'. First enounced by Wolff (1892), in its modern formulation the trajectorial theory states that the principal material directions of a given volume of cancellous bone are aligned with the principal stress trajectories generated from physiological loading, but only at spatial scales at which cancellous bone can be treated as a continuous material (Cowin 2001). The principal material directions describe the directions in which a volume of cancellous bone is most and least stiff, whereas (continuumlevel) principal stress trajectories describe how compressive and tensile forces are distributed throughout a material under a particular loading regime. As also reviewed in Part I, the principal 
91 material directions of a given volume of cancellous bone are closely aligned with its principal

92 fabric directions, that is, the directions of strongest and weakest alignment of trabeculae (Kabel et al. 1999; Odgaard et al. 1997; Turner et al. 1990; Ulrich et al. 1999). This effectively means that the architectural fabric of cancellous bone parallels the principal stress trajectories during the normal use of a bone. Such a correspondence has been demonstrated to occur in a wide variety of instances, again by both experimental (Biewener et al. 1996; Lanyon 1974; Su et al. 1999) and theoretical (Beaupré et al. 1990; Carter et al. 1989; Currey 2002; Gefen \& Seliktar 2004; Giddings et al. 2000; Hayes \& Snyder 1981; Jacobs 2000; Jacobs et al. 1997; Koch 1917; Miller et al. 2002; Pauwels 1980; Rudman et al. 2006; Sverdlova 2011; Vander Sloten \& Van der Perre 1989) studies of locomotion.

In the aforementioned theoretical studies, the general approach was the same. That is, given a continuum-level model of the bone, apply a loading regime that reflects in vivo physiological conditions (often derived from empirical measurements), calculate the resulting principal stress trajectories and then compare them to observations of cancellous bone architecture. It stands to reason that, if the trajectorial theory is true, the approach will also hold in reverse. Here, it is hypothesized that if one constructs a continuum-level model of a whole bone and seeks to determine the loading regime(s) in which principal stresses align with observed cancellous bone architecture, the resulting loading regime(s) should be physiologically realistic. It is also hypothesized that this 'reverse trajectorial approach', when framed in the context of a whole musculoskeletal system (such as a limb), should result in a physiologically realistic posture used during normal activity. If these predictions hold true, then this has the potential to provide new insight into understanding posture and locomotor biomechanics in extinct species, such as nonavian theropod dinosaurs, a group for which much interest surrounds their manner of locomotion (Hutchinson \& Allen 2009).

The present study aimed to test the above hypotheses, and thereby investigate the validity of the reverse approach. It focused on an extant theropod species, the chicken (Gallus gallus), as a generalized representative for all extant, ground-dwelling birds, for which much knowledge about terrestrial locomotor biomechanics exists. By integrating musculoskeletal and finite element modelling with observations of cancellous bone architecture, this study asked the question: "in 
122 what posture of the hindlimb do principal stresses align with observed cancellous bone

123 architecture, and is this posture consistent with empirical observations?" In testing the reverse 124 approach with a modern theropod and assessing its validity, the approach may then be applied to 125 extinct, non-avian theropods, as will be done in Part III (Bishop et al. in review-a). Additionally, 126 the results of the present study can also demonstrate how applicable this approach may be for 127 understanding locomotor biomechanics in extinct tetrapod vertebrates in general.

\section{II.3 Materials and Methods}

131

\section{II.3.1 The overall approach}

The concept of using cancellous bone architectural patterns to derive in vivo loading regimes is not new. However, previously developed approaches (Bona et al. 2006; Campoli et al. 2012; Christen et al. 2013a; Christen et al. 2015; Christen et al. 2013b; Christen et al. 2012; Fischer et al. 1995; Zadpoor et al. 2013) are so different from that of the present study, or indeed are likely not applicable to extinct species, that an examination of these approaches will be left to the Discussion. In the present study, the approach of identifying the loading regimes and hindlimb locomotor biomechanics that reflected observed cancellous bone architecture was a repetitive one, which may be summarized as follows. For a given test posture, the forces and moments involved were first calculated using a musculoskeletal model, assuming a quasi-static situation, which were then transferred to a set of finite element models to calculate principal stress trajectories in the femur, tibiotarsus and fibula. These stress trajectories were then compared to the observed cancellous bone fabric in each bone, as reported in Part I of this series. The amount of correspondence between stress trajectories and cancellous bone fabric, and where this occurred, was then used to guide the set-up of a new test posture. Starting from a general avian mid-stance posture, the process was repeated until new posture attempts did not produce improvement in overall correspondence compared to the previous best posture (they either produced similar or poorer levels of correspondence); at this point it was judged that no further significant improvement was likely to be gained. This repetitive procedure involved a 
152 combination of both manual and semi-automated techniques (detailed below), and in total took an

153 estimated 350 hours to perform.

154

155 The above procedure resulted in a single 'solution posture' that best reflected as much of the 156 observed cancellous bone architecture as possible, across all three bones. In seeking a single 157 posture, this study therefore sought the posture that engendered the greatest amount of

158 remodelling stimulus in cancellous bone, to which the bones responded and adapted their 159 architecture. Since bone remodelling is more responsive to repetitive, dynamic loading that 160 produces greater peak strains, as well as higher strain rates (Lanyon 1996; Turner 1998), the 161 movements in dynamic locomotion will presumably exert a strong influence on cancellous bone 162 architecture in limb bones. It was assumed here that the loading regime during mid-stance in 163 normal locomotion would be important for the determination of the observed cancellous bone 164 architecture. This is because the magnitude of the ground reaction force (GRF) is substantial at 165 around mid-stance in a wide range of animals, even if this is not when the absolute highest forces 166 are experienced across the stance phase (Alexander 1977; Andrada et al. 2013; Bishop et al. 167 2018; Blob \& Biewener 2001; Bryant et al. 1987; Butcher \& Blob 2008; Gosnell et al. 2011; 168 Hutchinson 2004; Ren et al. 2010; Rubenson et al. 2011; Sheffield \& Blob 2011; Witte et al. 169 2004). Measured in vivo joint reaction forces are also high at around this point in the stance 170 (Bergmann et al. 2001; Bergmann et al. 1999; Page et al. 1993; Taylor \& Walker 2001), as are 171 the reaction forces when calculated using biomechanical models (Giarmatzis et al. 2015; Goetz et 172 al. 2008; Lerner et al. 2015; Modenese \& Phillips 2012). Hence, a general avian mid-stance 173 posture was used as an initial starting point in the modelling process; this posture was not based 174 on any one species, but rather represented a qualitative 'average' of avian postures that have been 175 reported in the literature.

176

177 Several simplifications or assumptions were necessary throughout the modelling and simulation 178 process. These could have been avoided or refined if only extant theropods were the ultimate 179 focus of the study. However, as the approach outlined here needed to be applicable to extinct, 180 non-avian theropods as well, any limitations inherent to non-avian theropods, such as absence of 181 data concerning soft tissues (i.e., muscles, tendons, ligaments, cartilage, menisci) also had to be 182 observed in the chicken models and simulations. Thus, when there is good evidence of a feature 
183 or constraint in both the extinct and extant species, the attempt has been made to be a specific as

184

185

186

187

188

189

190

191

192

193

194

195

196

197

198

199

200

201

202

203

204

205

206

207

208

209

210

211

212

213

possible; however, when faced with considerable uncertainty or ambiguity, a more relaxed, generalized approach was taken. Not only does this tend to invoke fewer assumptions (i.e., model simplicity), but it also enables greater consistency across species for the sake of comparison.

All scripts, models and data used are held in the Geosciences Collection of the Queensland Museum, and are available upon request to the Collections Manager.

\section{II.3.2 Skeletal geometry acquisition}

This study focused on a single extant theropod species, the chicken (Gallus gallus), to explore the validity of the reverse approach. Chickens are a good generalized representative of extant, ground-dwelling birds, and much knowledge exists about their terrestrial locomotor biomechanics (Carrano \& Biewener 1999; Grossi et al. 2014; Muir et al. 1996; Rose et al. 2016). Furthermore, as demonstrated in Part I of this series (Bishop et al. in review-b), the cancellous bone fabric of chickens is quite typical of extant, ground-dwelling birds; in quantitative comparisons, chickens fall well within the region of space occupied by birds on streoplots of fabric directions. Thus, given the logistical constraints of time and resources, the chicken was deemed a good choice of species upon which to base the present study.

The models developed here were based on a $1.56 \mathrm{~kg}$ adult female chicken (white leghorn breed), which was studied previously by Bishop et al. (2018). This specimen was different to the two specimens that were investigated in Part I, on account of logistical reasons. The intact carcass was subject to X-ray computed tomographic (CT) scanning (Siemens Somatom Definition AS+, $120 \mathrm{kV}$ peak tube voltage, $255 \mathrm{~mA}$ tube current, $1000 \mathrm{~ms}$ exposure time, $0.367 \mathrm{~mm}$ pixel resolution, $0.2 \mathrm{~mm}$ slice thickness), and the resulting scans were segmented in Mimics 17.0 (Materialize NV, Belgium) via a combination of manual and automatic techniques. This produced an initial surface mesh for each bone, which was smoothed in 3-matic 9.0 (Materialize $\mathrm{NV}$, Belgium), and then refined to produce a more isoparametric mesh in ReMESH 2.1 (Attene \& Falcidieno 2006; http://remesh.sourceforge.net/). An isoparametric mesh is one in which the 
214 comprising triangles are all approximately equilateral in shape, and all of similar size. This is

215 important for the generation of a volume mesh for use in finite element analyses, because the 216 quality of the volume mesh is dependent on the quality of the surface mesh from which it is

217 derived (Wroe et al. 2007).

218

219 Refined surface meshes were produced for the femur, tibiotarsus, fibula and tarsometatarsus

220 (including metatarsal I), as well as the pelvis, sacrum and caudal vertebrae. These meshes were

221 used in the creation of the musculoskeletal model and their derived volume meshes were used in

222 the finite element model, facilitating complete node-to-node correspondence between the two

223 modelling environments. Despite the patella and tarsal sesamoid being present in the chicken,

224 they were not included in the development of the models, both for the sake of simplicity and also

225 to maintain consistency with models developed for non-avian theropods (in Part III), which lack

226 these bones. They did, however, help inform the construction of lines of action of muscles that

227 crossed the knee and ankle joints in the musculoskeletal model. In light of recent advances in

228 understanding of patellar mechanics in extant birds (Allen et al. 2017; Regnault et al. 2017),

229 future studies may be able to take the patella into account, although this would reduce

230 comparability between models of extant birds and non-avian theropods, the latter of which lacked 231 patellae.

232

233

234

\section{II.3.3 Musculoskeletal model development}

235

236

A musculoskeletal model of the right hindlimb of the chicken was constructed in NMSBuilder

237 (Martelli et al. 2011; Valente et al. 2014) for use in OpenSim 3.0.1 (Delp et al. 2007), and is

238 shown in Fig. 1. It comprised 12 degrees of freedom and 38 musculotendon actuators.

\section{II.3.3.1 Definition of joints}

243 The pelvis, sacrum and caudal vertebrae were fixed relative to each other and relative to the global reference frame, forming a single 'pelvis' segment. They were oriented such that a line 
245 through the neural canal of the anterior sacral vertebrae was horizontal and the postacetabular

246 pelvis sloped ventrally, comparable to the orientation of the pelvis of ground-dwelling birds

247 during stance and gait (Andrada et al. 2013; Gatesy 1999a; Rubenson et al. 2007). Although the

248 orientation of a bird's pelvis can vary during the stride and across different speeds of locomotion

249 (Abourachid et al. 2011; Gatesy 1999a; Rubenson et al. 2007), as a modelling simplification the

250 position or orientation of the pelvis segment, defined by six of the 12 model degrees of freedom

251 (three translational, three rotational), was fixed in all simulations.

252

253 The hip joint was modelled as a ball-and-socket joint with three degrees of freedom, namely

254 flexion-extension, adduction-abduction and long-axis rotation. The three axes of rotation were

255 initially parallel to the axes of the global coordinate system $(+x$ is anterior, $+y$ is medial, $+z$ is

256 dorsal), and the order of rotation was flexion-extension, followed by adduction-abduction,

257 followed by long-axis rotation. The centre of the joint in the femur was determined by fitting a

258 sphere to the femoral head in 3-matic, and the centre of the joint in the acetabulum was

259 determined by fitting a sphere to the concave articular surface in 3-matic. The femur was then

260 positioned relative to the pelvis such that the joint centres of the femur and acetabulum were

261 coincident. The 'neutral' orientation of the femur with respect to the pelvis (i.e., where all hip

262 joint angles are zero) was such that the standard anatomical directions for the bone were set

263 parallel to the axes of the global coordinate system $(+x$ is anterior, $+y$ is medial, $+z$ is proximal).

264 The neutral orientations for all bones distal to the femur were set by how they articulated with

265 their neighbouring proximal bone.

266

267 For simplicity, the knee joint was modelled with a single degree of freedom representing flexion-

268 extension, although it is acknowledged that in reality the avian knee is also capable of significant

269 abduction-adduction and long-axis rotation movement (Kambic et al. 2014; Rubenson et al.

270 2007). No translation of the flexion-extension axis was permitted (i.e., it was fixed relative to the

271 femur), neither was any relative movement between the tibiotarsus and fibula. The orientation

272 and position of the flexion-extension axis relative to the femur, tibiotarsus and fibula, and the

273 orientation and position of the tibiotarsus and fibula relative to the femur, was determined

274 manually. This ensured that there was realistic alignment and movement of the bones across the

275 physiological range of flexion-extension. For example, the tibiofibular crest of the lateral femoral 
276 condyle followed the space between the tibiotarsus and fibula at high flexion angles; no bone

277 interpenetration occurred at any orientation; and the amount of space between the tibiotarsus and

278 femur, and between the fibula and femur, remained fairly constant across the range of motion

279 (i.e., conservation of volume of the intervening soft tissues). Additionally, the alignment of the

280 bones was compared to their in situ orientations in the left and right limbs of the intact carcass, as

281 observed from the CT scans. Asymmetry in the size and shape of the distal femoral condyles

282 inherently meant that when the femur was in the neutral orientation, the knee joint axis was

283 angled slightly mediolaterally in the coronal plane (Fig. 1E). Consequently, this also meant that

284 in the neutral orientation, the distal end of the tibiotarsus and fibula were angled in towards the

285 body midline (Fig. 1B,E).

286

287 Given the likely sizeable quantities of cartilage and menisci in the knee joint of extinct, non-avian

288

289

290

291

292

293

294

295

296

297

298

299

300

301

302

303

304

305

306 theropods (e.g., Bonnan et al. 2010; Bonnan et al. 2013), and the fact that the present study needed to be wholly consistent with any modelling limitations inherent to non-avian theropods, it was felt that this representation of the knee would be more reliable than a strictly objective, geometry-based definition of the joint axis (Brassey et al. 2017; Hutchinson et al. 2005;

Hutchinson et al. 2008; although these studies did include space for soft tissues). This is because such a definition is only based on the available bony geometry, which may not fully reflect the actual range of possible joint movement. Moreover, such a definition only uses half of the contributing joint surfaces, for example using the femur whilst ignoring the tibiotarsus and fibula. Differences in how the knee joint is defined would be expected to have an influence on both the orientation of the knee joint axis relative to the bones and the neutral orientation of the limb.

The ankle and metatarsophalangeal joints were both modelled with a single flexion-extension degree of freedom, although as for the knee it is acknowledged that this is a simplification of reality (Kambic et al. 2014). As for the knee joint, no translation of the flexion-extension axis was permitted in either joint; the ankle axis was fixed relative to the tibiotarsus, and the metatarsophalangeal axis was fixed relative to the tarsometatarsus. The flexion-extension axis of the ankle joint was determined in 3-matic by fitting a cylinder to the outer margins of the articular surfaces of the tibiotarsus, with the axis of the cylinder taken to be the axis of movement. The flexion-extension axis of the metatarsophalangeal joint was taken to be parallel to the $y$-axis 
307 308

309

310

311

312

313

314

315

316

317

318

319

320

321

322

323

324

325

326

327

328

329

330

331

332

333

334

335 336

when the limb was in a neutral orientation. Metatarsal I was fixed relative to the tarsometatarsus, and digit I was not modelled. Care was taken to ensure that bone interpenetration did not occur at these joints as well, over the range of joint motion typically reported for avian terrestrial locomotion in the literature.

The pes (digits II-IV) was modelled as a rectangular prism, parallel to the axes of the global reference frame in the neutral limb orientation, as done by Hutchinson et al. $(2005,2008)$. This was not only for model simplicity, but also because of the uncertainty surrounding the topology and degree of differentiation of pedal muscles in non-avian theropods (Carrano \& Hutchinson 2002; Hutchinson 2002). Hence, for consistency, these modelling limitations inherent to nonavian theropods were also observed in the chicken model. The length of the prism was set as the total length of digit III, and the width set as the mediolateral width of the distal tarsometatarsus, across the condyles.

\section{II.3.3.2 Definition of muscle and ligament anatomy}

A total of 34 musculotendon actuators were used to represent muscles in the model; an additional four actuators were used to represent the medial and lateral collateral ligaments of the knee and ankle, thus allowing the possibility of 'passive' forces to be included. The origins and insertions of the actuators in the model (Table 1) were derived from first-hand observations made during dissections (from four individuals in total), as well as comparison to the published literature (e.g., Hudson 1937; Hudson et al. 1959; Paxton et al. 2010), and were placed as near as possible to the centroid of the area of attachment in each case. The 3-D course of each actuator from origin to insertion was constrained to follow anatomically realistic paths as observed during dissections and reported in the literature. This was achieved through the placement of a number of intermediate 'via points' (Delp et al. 1990) along the course of the actuator. Only the minimum number of via points was used to achieve realistic paths, across the whole physiological range of motion. 
337 For the purposes of the current study, a number of simplifications were made regarding the

338 representation of some of the muscles:

339 1. The popliteus was not included in the model, for it runs between the proximal tibiotarsus and fibula. Since relative movement between the two bones was not modelled here, inclusion of the popliteus is unnecessary.

2. The plantaris was not included in the model, because it runs from the proximal tibiotarsus to the medial aspect of the tibial cartilage surrounding the ankle; it was therefore considered unlikely to play a significant role in load bearing, and thus load transmission to the bones. For a similar reason, the secondary attachment of the fibularis longus (FL) to the tibial cartilage was also not modelled.

3. On account of its small size, similar line of action to, and common insertion with, the obturatorius medialis (OM), the obturatorius lateralis was not modelled: a single musculotendon actuator was deemed sufficient to represent the two muscles.

4. Both parts of the flexor cruris lateralis (pars pelvica, FCLP; pars accessoria, FCLA) were modelled with separate musculotendon actuators. At the point where the FCLA diverges from the FCLP, the actuators went their own separate way towards their respective insertions, but proximal to this, they took on the same line of action towards the origin on the pelvis. A similar approach was used for modelling the two heads of the tibialis cranialis (caput femorale, TCF; caput tibiale, TCT).

5. The flexor hallucis brevis and extensor hallucis longus were not modelled, because they run from the tarsometatarsus to the ungual of digit I; as there was no degree of freedom that these two muscles could influence in the model, they were unnecessary.

6. As noted above, there is considerable uncertainty surrounding the topology and degree of differentiation of many of the digital flexor and extensor muscles in non-avian theropods (Carrano \& Hutchinson 2002; Hutchinson 2002). The representation of these muscles in the chicken model was consequently simplified, to maintain consistency with non-avian theropod models, but also because the pes was modelled as a single unit. The flexor digitorum longus (FDL) and flexor hallucis longus (FHL) were modelled separately, but the deep digital flexors were represented by a single musculotendon actuator ('other digital flexors', ODF), which grossly reflected the lines of action of the individual muscles. Likewise for the extensors, the 
367

368

369

370

371

372

373

374

375

376

377

378

379

380

381

382

383

384

385

386

387

388

389

390

391

392

393

394

395

396

397

extensor digitorum longus (EDL) was modelled separately, but the deep digital extensors were represented by a single musculotendon actuator ('other digital extensors', ODE).

7. Owing to the simplified representation of the deep digital flexors, the main insertion of the FL was extended to the ventral aspect of the pes segment.

These modelling simplifications were not expected to have any significant influence on the loading conditions experienced by the femur, tibiotarsus or fibula.

The 38 musculotendon actuators so modelled here provided the forces necessary to counter collapse of the hindlimb during the simulation of a given test posture. Whilst the maximum force able to be produced by each muscle (or resisted by each ligament) could be estimated from empirical anatomical data (Calow \& Alexander 1973; Hutchinson et al. 2015; Lamas et al. 2014), this is obviously not possible in the case of extinct, non-avian theropods. As such, for the sake of simplicity and consistency across extinct and extant species, all musculotendon actuators were assigned the same maximum force, $30.597 \mathrm{~N}$, equal to two times body weight (BW). A value of 2 BW was chosen because some muscles would have undoubtedly been capable of exerting forces of that magnitude, or greater, as is the case in other animals (Anderson \& Pandy 1999; Charles et al. 2016; Hutchinson et al. 2015; O'Neill et al. 2013; Smith et al. 2006). With all actuators having the capacity to exert forces of that magnitude, there was ample force for the actuation of each degree of freedom, obviating the need for reserve actuators (but see Section II.3.4.3 below).

\section{$\underline{\text { II.3.3.3 Definition of segment mass properties }}$}

As a means to estimating the mass properties of each limb segment in the musculoskeletal model, the flesh surrounding each limb bone was segmented from the carcass CT scans in Mimics to produce a series of surface meshes. Using the computer-aided design software Rhinoceros 4.0 (McNeel, USA), each flesh mesh was then repositioned in space to align it with the underlying bone(s) in their neutral orientation. Additionally, the thigh segment flesh was retro-deformed to fit the pelvis and femur in the neutral pose, and care was taken to ensure that the net change in volume was negligible; this process was accomplished in Rhinoceros using the 'cage edit tool', a form of host mesh warping (Fernandez et al. 2004). The mass and centre of mass (COM) of each 
398

399

400

401

402

403

404

405

406

407

408

409

410

411

412

413

414

415

416

417

418

419

420

421

422

423

424

425

426

427

428

segment was then able to be calculated in NMSbuilder, assuming a bulk density of $1000 \mathrm{~kg} / \mathrm{m}^{3}$. The total mass of the right hindlimb in the model was $0.159 \mathrm{~kg}$, and therefore the mass of the remaining body was $1.401 \mathrm{~kg}$; this was designated as the mass of the pelvis segment in the model. Given the data reported by Allen et al. (2013), the combined COM of the whole body, minus the right leg, in their geometric model of a chicken was $0.076 \mathrm{~m}$ anterior to the hip joint. Scaling isometrically (via femur length) to the chicken specimen modelled here, the COM is $0.068 \mathrm{~m}$ anterior to the hip; this was taken to be the location of the COM of the pelvis segment in the musculoskeletal model. Since the orientation of the pelvis segment was fixed in all simulations, and all simulations were quasi-static, the only moment produced by the pelvis segment would be that by virtue of its weight, and consequently the dorsoventral position of the pelvis segment COM would not matter. As such, the dorsoventral position of the COM of the pelvis segment was assumed to be level with the hip. Moments of inertia for each segment were not calculated, on account that the simulations performed in this study were quasi-static only.

\section{II.3.4 Musculoskeletal simulations}

\section{II.3.4.1 Deriving a test posture}

Based on the argument presented in Section II.3.1 above, a general mid-stance posture was used as an initial starting posture, which was then modified in subsequent modelling attempts. It was based on comparison to the kinematic data previously reported for ground-dwelling birds (e.g., Abourachid \& Renous 2000; Gatesy 1999a; Grossi et al. 2014; Reilly 2000; Rubenson et al. 2007; Stoessel \& Fischer 2012): hip extension of $-30^{\circ}$ below horizontal, hip abducted $5^{\circ}$ from midline, hip rotated $20^{\circ}$ externally, knee flexed $93^{\circ}$ from neutral position, ankle flexed $46^{\circ}$ from neutral position, metatarsophalangeal joint extended $16^{\circ}$ from neutral position. The modification of a given test posture to produce a new posture at the start of a new modelling attempt followed 
429 hierarchical priorities: hip extension angle $>$ knee angle $>$ ankle and metatarsophalangeal angles,

430 with the metatarsophalangeal angle set so as to position the pes segment flat on the ground (i.e.,

431 parallel to the $x-y$ plane). Each posture was also constrained by thee basic criteria:

432 1. No interpenetration occurred between any bones, including those of the pelvis.

433 2. The centroid of the pes segment, taken to be the location of the centre of pressure (COP) of the 434 GRF (see below), was underneath the whole-body COM in the $x$ - $z$ plane. This constraint

435 predominantly affected the knee, ankle and metatarsophalangeal joint angles, and was

436 necessitated by the fact that the applied ground reaction force in the simulations was vertically

437 oriented (see below).

438 3. The mediolateral step width, defined as twice the distance from the centroid of the pes 439 segment to the body midline, was less than $15 \%$ of the posture's hip height, defined as the 440 vertical distance from the hip joint centre to the base of the pes segment. This constraint 441 predominantly affected the hip adduction-abduction and long-axis rotation angles, and was 442 based on the results of Bishop et al. (2017b).

443

444

445

446

447

448

449

450

451

452

453

454

455

456

457

458

\section{II.3.4.2 External forces}

In the present study, a given test posture was analysed as a quasi-static system. Dynamic effects such as segment accelerations were not considered, as this requires additional information and assumptions about movement, which are currently unknown for extinct, non-avian theropods. (Furthermore, incorporating dynamic effects might not actually lead to a marked change in model results, e.g., Anderson \& Pandy 2001; Rankin et al. 2016.) Hence, the only acceleration in the simulation was that due to gravity, of magnitude $1 \mathrm{BW}$. In order for static equilibrium to be maintained, and also to refrain from using residual actuators of the six degrees of freedom at the pelvis, this necessitated the applied GRF to be vertical and also of magnitude $1 \mathrm{BW}$ (Fig. 2). This in turn required one of the following three scenarios to also be true: 
459

460

461

462

463

464

465

466

467

468

469

470

471

472

473

474

475

476

477

478

479

480

481

482

483

484

485

486

487

488

489

1. The centroid of the pes segment (taken as the COP of the GRF) must be directly underneath the whole-body COM, in both the $x$ and $y$ directions. However, the whole-body COM is on (or almost on) the body midline, meaning that in such a scenario the pes is also on the body midline. This is posturally inaccurate, because theropods employ non-zero step widths across most speeds (Bishop et al. 2017b).

2. The centroid of the pes segment is not directly underneath the whole-body COM, instead having a non-zero step width. This is more posturally accurate, but static equilibrium will not be achieved unless:

(a) The COP is moved away from the centroid of the pes and retained directly under the wholebody COM. This is more speculative however, because empirical data on the path of the COP in modern bipeds shows that it remains close to the centre of the foot, not straying too far laterally or medially away from the foot midline (Schaller et al. 2011; Winter 2009).

(b) The COP is kept at the centroid of the pes, and an additional moment about the $x$-axis is applied to the pes. This moment is equal to the product of BW and the mediolateral distance between the COM and COP:

$$
M_{x}=m \cdot g \cdot\left(\mathrm{COP}_{y}-\mathrm{COM}_{y}\right),
$$

where $m$ is body mass and $g$ is the acceleration due to gravity, $9.81 \mathrm{~m} / \mathrm{s}^{2}$. This is physiologically implausible however, as in reality the feet can only be capable of applying a moment about the vertical $(z)$ axis, the so-called 'free moment'.

Hence, regardless of which scenario is used, some amount of accuracy must be lost in order for static equilibrium to be achieved and the simulation to be solved. The present study followed scenario 2(b), in order to maintain postural accuracy (Fig. 2). An additional moment about the $y$ axis $\left(M_{y}\right)$ was also applied to the pes to account for minute positional discrepancies between the $x$-coordinates of the COP and COM (i.e., when the COP was not exactly underneath the COM), but this never amounted to more than $0.011 \mathrm{Nm}(<1 \%$ of the product of body weight and COM height) in any of the simulations performed.

That the applied GRF in the simulations was vertical is appropriate in the context of the current study for two reasons. Firstly, in a wide range of animals including theropods the GRF is approximately vertical, in the sagittal plane, at around mid-stance (e.g., Bishop et al. 2018; Hutchinson 2004). Secondly, in a wide range of animals including theropods the GRF is largely 
490

491

492

493

494

495

496

497

498

499

500

501

502

503

504

505

506

507

508

509

510

511

512

513

514

515

516

517

518

519

520

vertical at the instance of peak net GRF (especially in more 'running-like' gaits), and this instance also occurs at around mid-stance (e.g., Bishop et al. 2018). However, when the GRF is at its most vertical in the sagittal plane, or when it is at maximum magnitude, it is almost always never $1 \mathrm{BW}$ in magnitude; it is sometimes a little lower, but most often it is higher, and sometimes much higher, than $1 \mathrm{BW}$. This is not a problem for the current study, because principal stress trajectories do not reflect the absolute magnitudes of applied forces, only their relative magnitudes and directions, provided that deformation remains within the elastic range (Beer et al. 2012). Moreover, in having the GRF as $1 \mathrm{BW}$ in magnitude, this also facilitates sizeindependent comparisons across postures and across species following simulation.

\section{II.3.4.3 Simulation and calculation of internal forces and moments}

Once a test posture was established and the GRF (and associated moments) was applied, the forces developed by the musculotendon actuators to resist limb collapse were calculated in OpenSim. Although 34 muscles were represented in the musculoskeletal model, not all of them would be active and exerting force at around the mid-stance of a stride. Thus, some muscles were set to be inactive in the simulations (Fig. 2, Table 2). Which muscles were set to be inactive was determined through reference to published electromyography data for birds (Gatesy 1990; Gatesy 1994; Gatesy 1999b; Jacobson \& Hollyday 1982; Marsh et al. 2004; Roberts et al. 1998).

Muscles that are active only in the swing phase, or active in the stance phase but only at the very beginning or end, were considered inactive. If no data existed for a particular muscle, the following line of reasoning was employed. If the muscle belonged to the same functional group as another muscle that had been investigated (e.g., femorotibialis externus, FMTE;

puboischiofemoralis lateralis, PIFL), its activity was assigned based on the recorded muscle.

Failing that, if the muscle was considered unlikely to be involved in limb support at around midstance (e.g., ankle flexors, digital extensors, OM, IFI), it was considered inactive. If its activity still remained equivocal after that, then it was included in the model and deemed to be active, to be conservative. All four collateral ligament actuators were also included, to allow for passive forces to occur. These were modelled simply as linear 'reserve' actuators without incorporation of slack length or elasticity. 
522 On account of the unknowable properties of muscle and ligament in extinct theropods, intrinsic

523 force-length-velocity relationships were ignored for all musculotendon actuators in the

524 simulations. That is, the actuators simply modelled the application of a force along a line of

525 action set by the actuator geometry, defined in Section II.3.3.2 above. Hence, the moment $M_{i}$ a

526 given actuator exerted about a given degree of freedom $i$ was equal to

527

$$
M_{i}=a \cdot F_{\max } \cdot r_{i},
$$

528 where $F_{\max }$ is the maximum force capable of being produced (set at $2 \mathrm{BW}$ ), $r_{i}$ is the moment arm

529 of the actuator and $a$ is the activation of the actuator, which can vary between 0 and 1 . The forces

530 developed in each musculotendon actuator were calculated using the static optimization routine

531 of OpenSim, which solved the statically indeterminate problem of force distribution by

532 minimizing the sum of squared activations across the actuators (Pedotti et al. 1978; Rankin et al.

533 2016). It was found that in no simulation did the activation of any musculotendon actuator ever

534 approach 1; indeed, activations rarely exceeded 0.5. Coupled with the omission of intrinsic force-

535 length-velocity relationships, this prevented nonlinearities from occurring in the static

536 optimization routine, further facilitating size-independent comparisons across postures and across

537 species post analysis. Due to the simplified representation of the pes segment and the muscles

538 that cross the metatarsophalangeal joint, a reserve actuator was also applied to the

539 metatarsophalangeal joint in the static optimization, with a maximum output set at 1,000 $\mathrm{Nm}$

540 (Fig. 2). This high value provided ample control of the metatarsophalangeal joint, and helped

541 reduce excessively high and unrealistic recruitment of the few modelled musculotendon actuators

542 that crossed the joint (FDL, ODF and FL). Across the range of postures tested, the reserve

543 actuator never provided a moment greater than $0.41 \mathrm{Nm}(<12 \%$ of the product of body weight

544 and COM height). In addition to the actual calculated forces, the line of action of all

545 musculotendon actuators was also extracted from the posture, using the MuscleForceDirection

546 plugin for OpenSim (van Arkel et al. 2013). Following the calculation of muscle and ligament

547 forces, joint forces and moments were extracted using the JointReaction tool in OpenSim (Steele

548 et al. 2012). All forces were extracted and expressed in the global coordinate system.

II.3.5 Finite element simulations 
552

553 Two finite element simulations were performed for each test posture in ANSYS 17.0 (Ansys, 554 Inc., USA), one of the femur and one of the tibiotarsus + fibula. The loads applied in these

555 simulations were exactly the same as those calculated in the musculoskeletal simulations.

556 Furthermore, the nodes on each bone to which muscle or ligament forces were applied in the

557 finite element simulations were the exact same nodes to which the musculotendon actuators

558 attached in the musculoskeletal simulations. This ensures complete correspondence between the

559 two sets of simulations.

560

561

562 II.3.5.1 Geometry

563

564 The relative positions and orientations of each bone in the musculoskeletal simulations were

565 maintained exactly in the finite element simulations. In addition to the modelling the focal bone

566 (or bones) of interest, two extrinsic structures were created to represent the adjacent articulating

567 bones, to more realistically model the distribution of joint forces (Fig. 3A,B). For the femur

568 simulation, an acetabulum structure (derived from the pelvis surface mesh) and proximal crus

569 structure (derived from the tibiotarsus and fibula surface meshes) were created. For the

570 tibiotarsus + fibula simulation, a distal femur structure (derived from the femur surface mesh) and

571 proximal tarsometatarsus structure (derived from the tarsometatarsus surface mesh) were created.

572 These structures were generated simply by trimming their parent surface meshes down to the

573 immediate area involved in the joint articulation, using a combination of Rhinoceros and 3-matic.

574 Additionally, in the proximal crus structure, the geometry was modified distally, well away from

575 the articular areas, to fuse the tibiotarsus and fibula together, limiting movement between the two

576 during the simulations.

577

578 In order to model the distribution of joint forces more realistically and evenly across opposing 579 joint surfaces, the intervening soft tissues that occur between a focal bone and its neighbouring 580 bone in life were modelled as a single volume (Fig. 3). A single, homogenous entity was chosen 581 to represent these joint soft tissues (e.g., cartilage, menisci) in the current study, as the anatomy 582 of such tissues is unknown for extinct, non-avian theropods. Moreover, this modelling 
583 simplification makes the analyses more tractable for the current study, instead of involving more 584 complex, non-linear behaviours and contact formulations. The volume of soft tissues for each of 585 the hip, knee and ankle joints was produced by connecting up the closest parts of the articular 586 surfaces of the bones involved, using the 'loft' tool in Rhinoceros to create an initial mesh, which 587 was then smoothed and remeshed in 3-matic. In addition to more realistically modelling joint 588 load distribution, the approach used here also allowed for boundary conditions (restraints) to be 589 moved away from the bone (or bones) of interest, reducing the incidence of artifacts in the model 590 results (Saint-Venant's principle: Dumont et al. 2005; Gilbert et al. 2016; McHenry et al. 2007). It 591 is conceptually similar to the approach employed by Phillips and co-workers in their finite 592 element modelling of human limb bones (Geraldes et al. 2016; Geraldes \& Phillips 2014; Phillips 593 et al. 2015), although the actual formulations involved are markedly different.

594

Volume meshes for finite element analysis were generated from the surface meshes of each bone 596 and soft tissue entity in 3-matic. All volume meshes were composed exclusively of low-order (4node) tetrahedral elements. Meshes composed of high-order (10-node) elements may produce more accurate results than those composed of low-order elements, but this discrepancy decreases with a greater number of elements used (Bright \& Rayfield 2011; Dumont et al. 2005).

600 Furthermore, considering the relatively simple geometry of the bones being modelled here, any 601 such discrepancy was considered to be minimal. In producing the volume meshes, the maximum tetrahedral edge length was constrained, so as to avoid the generation of tetrahedral elements of undesirably high aspect ratios, which can lead to inaccurate results. The maximum edge length for each entity was defined as being no more than double the mean edge length of the triangles in the parent surface mesh. The mean edge length of the surface mesh triangles was calculated as

$$
L=\sqrt{\frac{4 A}{\sqrt{3} n}},
$$

607 where $A$ is the total area of the surface mesh and $n$ is the number of comprising triangles in the 608 surface mesh. This assumes that the average triangle in the surface mesh is equilateral in shape ${ }^{1}$. 609 The total number of elements used across the various postures tested ranged from 803,508 to

\footnotetext{
${ }^{1}$ In an equiliateral triangle of edge length $L$, its area is given by $\frac{\sqrt{3}}{4} \cdot L^{2}$; setting this equal to average triangle area $\frac{A}{n}$ and solving for $L$ gives equation 3 .
} 
610822,322 in the femur simulation and from 986,280 to $1,005,550$ in the tibiotarsus + fibula

611 simulation. Although a convergence analysis was not conducted, it was considered that this was a

612 sufficient number of elements for the current study, given the relatively simple geometry of the

613 structures being modelled (Bright \& Rayfield 2011; Gilbert et al. 2016).

614

615 In the finite element simulations, the interfaces of adjacent contacting entities (e.g., hip soft

616 tissues and femur) were fixed relative to each other using a bonded contact formulation ANSYS,

617 such that they did not move or separate relative to each other. This facilitates seamless load

618 transmission from one entity to another. Bonded contact was also used to model the connection

619 between the fibula and fibular crest of the tibiotarsus, even though their respective interfaces

620 were not in actual direct contact.

621

622

623

\section{$\underline{\text { II.3.5.2 Material properties }}$}

624

625

All entities were modelled as solid, isotropic, linearly elastic materials. Three different materials

were defined for the entities being modelled (Table 3): bone, cartilage (for the hip and ankle soft

627 tissue entities) and a composite of the material properties of cartilage and menisci (for the knee

628 soft tissue entity). Extinct, non-avian theropods are inferred to have had menisci in their knee joints, based on their widespread occurrence in extant tetrapods, including birds and crocodilians (Chadwick et al. 2014; Haines 1942; Wink et al. 1989; Zinoviev 2010), but the actual morphology of these menisci remains speculative. This is one of the reasons for modelling all soft tissues in the knee joint as a single, homogenous entity, in addition to being a computational simplification. The material properties assigned for bone were conservatively estimated from the most common values reported for cortical bone in the literature (e.g., Currey 2002, and references cited therein; Erickson et al. 2002; Reed \& Brown 2001). The material properties for cartilage and menisci were also conservative estimates, derived from the literature (e.g., Currey 2002, and references cited therein; Kazemi et al. 2013; Stops et al. 2012).

In previous finite element studies, cartilage and menisci have been represented with a variety of 640 material behaviours, including isotropic and transversely isotropic linear elasticity, 
641 hyperelasticity, viscoelasticity and poroelasticity (Kazemi et al. 2013; Stops et al. 2012). The use

642 of isotropic, linearly elastic material behaviour in the present study is justified on the following

643 grounds. Firstly, as the analyses of the present study were quasi-static, the time (strain rate)

644 dependency of nonlinear material properties can be ignored with minimal error (Carey et al.

645 2014). Secondly, the precise kind of material behaviour, or material properties, is virtually

646 unknown for any archosaur (extinct or extant). Thirdly, assuming an isotropic, linearly elastic

647 material behaviour kept the model simple and minimally speculative, and also reduced the

648 computational cost of solving the finite element models.

649

650

A solid, isotropic, linearly elastic continuum representation was also necessitated for the bone

651

entities in the simulations. Not only is this because material properties (and any anisotropy

652

thereof) are unable to be determined for extinct theropods, but moreover anything other than this

653

representation could compromise the objectives of the current study. Specifically, the

654

introduction of any sort of structural or material heterogeneity, discontinuity or directionality will

655 influence the resulting principal stress trajectories. Since a key objective of this study was to

656 examine the spatial variation of the calculated principal stress trajectories in relation to cancellous

657 bone architecture, directionality needed to be a model output only, not a model input.

658

659

660

\section{$\underline{\text { II.3.5.3 Loads and restraints }}$}

661

662 For each simulation, four sets of loads were applied: muscle and ligament forces, joint forces, 663 joint moments and segment weight. As noted above, muscle and ligament forces were applied to 664 the same nodes as were involved in the musculoskeletal simulations. Additionally, a given 665 muscle or ligament force was evenly spread out over a number of nodes (generally around 20), 666 centred about the focal node, in order to reduce the incidence of artifacts in the model results. 667

668 Joint forces were applied to a focal bone via its neighbouring bones. Here, one neighbouring bone 669 was restrained in translation in all three axes, whilst the other was used to apply a joint force; the 670 joint force at the restrained end of the bone was provided by the reaction at the restraints, 671 transmitted back through the bone of interest. In both the femur and tibiotarsus + fibula 
672 simulations, the knee joint force was applied directly via the appropriate neighbouring bone

673 (proximal tibiotarsus + fibula and distal femur, respectively), with the other neighbouring bone

674 being restrained (acetabulum and proximal tarsometatarsus, respectively). In ANSYS, this

675 approach was implemented by using a 'remote force' (Fig. 3D). This is where a force is applied

676 to a specific entity, but via a remote point in space that is topologically attached ('scoped') to the

677 entity; when a force is applied to the remote point, the target entity gets pulled or pushed along

678 with it, along the line of action of the applied force. In ANSYS, this is accomplished by a set of

679 constraint equations that relate the degrees of freedom of an entity's nodes to the remote point;

680 one constraint equation exists for each node in the entity experiencing the remote force. The

681 location of the remote point in both the femur and tibiotarsus + fibula simulations was specified

682 as the location of the knee joint centre in the musculoskeletal model. This meant that the joint

683 force was applied properly, without introducing any moments into the system, because the net

684 force vector passed through the correct location in space, again ensuring complete

685 correspondence between the finite element and musculoskeletal simulations.

686

687 The knee joint moment was applied directly to the appropriate bone or bones, by applying it to

688 the surface or surfaces in contact with the knee soft tissues; for example, by applying it to the

689 distal femur in the femur simulation. This direct application was chosen, as opposed to the

690 moment being applied via a neighbouring bone, because the greater compliance of the knee soft

691 tissues would not allow full transmission of the moment to the bone or bones of interest. No hip

692 joint moment was involved, since the hip joint was modelled as a ball-and-socket joint, and thus

693 unable to resist moments. Whilst an ankle joint moment was calculated in the musculoskeletal

694 simulations, it was not able to applied in the tibiotarsus + fibula finite element simulations. This

695 is because of the close proximity of the ankle end of the tibiotarsus to the restraint, and thus the

696 restraint would greatly alter the transmission of any applied moment; this modelling deficiency

697 will be returned to in the Discussion (Section II.5.1).

698

699 The weight of the appropriate segment (e.g., thigh segment weight in the femur simulation) was

700 applied via a remote point that was scoped to the entire bone of interest. The location of the

701 remote point was set as the COM of the limb segment.

702 
$\underline{\text { II.3.5.4 Model solution }}$

705

706

All finite element simulations were solved as linear static systems in the Static Structural module

707

708

709

710

711

712

713

714

715

716

717

718

719

720

721

722

723

724

725

726

727

728

729

730

731

732

733

of ANSYS. Additionally, all simulations used inertia relief, which is a technique that is used to counter unbalanced forces, so as to produce no net acceleration of the model (Liao 2011). This is achieved through the application of an inertial force and moment to the model's centre. Although the musculoskeletal simulations described above were analysed under the assumption of static equilibrium, this does not exactly occur in finite element simulations due to non-rigid behaviour of the various entities. In particular, the soft tissue structures are highly compliant relative to the bone structures, and deformation of these soft tissue structures during simulation will lead to an imbalance of the applied forces. This has the potential to produce a positive-feedback loop where force imbalance leads to model acceleration, which leads to further deformation, which in turn leads to greater force imbalance, and so on. Ultimately, very large and unrealistic deformations occur, and calculated model results are unreliable. Thus, inertia relief was used to counter the initially very small imbalance in forces that results upon deformation of the model; for instance, in the femur simulation of the solution posture, the applied inertial force was

$$
\left(F_{x}, F_{y}, F_{z}\right)=\left(7.1725 \times 10^{-8}, 6.7934 \times 10^{-8},-1.1303 \times 10^{-6}\right) \mathrm{N},
$$

and the applied inertial moment was

$$
\left(M_{x}, M_{y}, M_{z}\right)=\left(1.7224 \times 10^{-6}, 3.3496 \times 10^{-6},-5.3604 \times 10^{-7}\right) \mathrm{Nm} .
$$

The very small magnitude of these adjustments justifies the use of this technique in the current study.

\section{II.3.6 Results analysis}

Upon performing the finite element simulations for a given test posture, the calculated stress tensor at each node in each bone entity was exported from ANSYS. A custom script in MATLAB 8.0 (MathWorks, USA) was then used to perform an eigenanalysis of the stress tensor data, producing the vector orientations of the principal stresses. Their 3-D trajectories were then 
734 visualized using this MATLAB script, as well as Rhinoceros. These trajectories, particularly of

735 the maximum principal stress $\left(\boldsymbol{\sigma}_{1}\right.$, usually signifying tension) and minimum principal stress $\left(\boldsymbol{\sigma}_{3}\right.$,

736 signifying compression) were visually (qualitatively) compared to the architectural patterns of

737 cancellous bone fabric reported for birds in Part I (Bishop et al. in review-b). As a further aid to

738 assessing the degree of correspondence between principal stresses and cancellous bone fabric, the

739 direction of $\boldsymbol{\sigma}_{3}$ in the femoral head and medial femoral condyle was quantitatively compared to

740 the mean directions of the primary fabric direction $\left(\mathbf{u}_{1}\right)$ for those parts of the femur in birds, also

741 as reported in Part I. As $\sigma_{3}$ is compressive, it stands to reason that this will show the greatest

742 correspondence with the architecture in the femoral head and medial femoral condyle, both of

743 which would be expected to be exposed predominantly to compressive joint loading. The

744 direction of $\sigma_{3}$ in the femoral head was taken to be the mean direction of vectors in the region of

745 a sphere of radius one-half of that fit to the entire femoral head (performed in 3-matic), and

746 positioned just under the surface of the bone, underneath where the hip force was received in the

747 finite element simulations. The direction of $\boldsymbol{\sigma}_{3}$ in the medial femoral condyle was taken to be the

748 mean direction of vectors in the region of a sphere of radius one-third of that fit to the condyle

749 (performed in 3-matic), and positioned in the anatomical centre of the condyle. Higher priority

750 was given towards improving correspondence in the femoral head over the medial condyle, since

751 hip angles are presumed to be more important for determining overall posture in bipeds; as the

752 most proximal joint (and with three degrees of freedom), the hip will have the greatest influence

753 on overall limb positioning (cf. Hutchinson \& Gatesy 2000), as well as the disposition of more

754 distal joints in the limb. Additionally, strict comparison between the mean directions of $\boldsymbol{\sigma}_{3}$ and $\mathbf{u}_{1}$

755 in the medial femoral condyle ignores the 'fanning' of $\mathbf{u}_{1}$ that occurs in this region of the bone

756 (see Part I), and hence is less legitimate.

757

758 Comparisons were made from the chicken finite element stress results to the architectural patterns 759 observed in ground-dwelling birds as a whole for two main reasons:

760 1. It has been shown that birds as a whole appear to demonstrate a largely consistent pattern of cancellous bone architecture in the femur, tibiotarsus and fibula (Part I). That is, the architectural patterns thus far observed can be described by a single 'archetype', about which there was specimen-specific (perhaps species-specific, pending greater sampling) variation. 
765

766

767

768

769

770

771

772

773

774

775

776

777

778

779

780

781

782

783

784

785

786

787

788

789

790

791

792

793

794

795

2. Cancellous bone architecture could not be extensively quantified in smaller birds such as chickens, owing to continuum level restrictions (relatively few trabeculae; see Part I). Nevertheless, where cancellous bone fabric was quantifiable in chickens, the results were close to the mean 'archetypal' value for birds overall (Part I).

As this series of studies is exploratory with a small sample size for each examined avian species (Part I), it is prudent (and conservative) to make comparisons to ground-dwelling birds as a whole, until such a time that significant interspecific differences can be demonstrated, in terms of both locomotor behaviour and cancellous bone architecture.

\section{II.3.7 Caveats}

Two points are worth noting about the overarching philosophy of the approach of the current study. Firstly, this study sought to determine a single posture, whose principal stress trajectories showed the greatest degree of correspondence to observed cancellous bone architecture in the femur, tibiotarsus and fibula. Cancellous bone, however, experiences many different loading regimes throughout the course of normal activity, each of which engenders a remodelling stimulus, and to which cancellous bone responds and adapts its architecture (Kivell 2016). This has been demonstrated in many previous computational theoretical studies, whereby no one loading regime will lead to replication of all of the observed architectural features in a bone; only when multiple loading regimes are considered can all of a bone's cancellous architecture be explained (Beaupré et al. 1990; Bona et al. 2006; Boyle \& Kim 2011; Carter \& Beaupré 2001; Carter et al. 1989; Coelho et al. 2009; Jacobs et al. 1997; Jang \& Kim 2008; Jang \& Kim 2010a; Jang \& Kim 2010b; Phillips et al. 2015; Sverdlova 2011; Tsubota et al. 2002; Tsubota et al. 2009; Turner et al. 1997). Therefore, in seeking a single posture that best reflects the observed cancellous bone architecture, the current study in fact searched for a 'characteristic posture', which is a time- and load-averaged posture across all loading regimes. This characteristic posture may or may not be an actual posture used at a particular instance in a particular behaviour. As argued above, however, the posture at around the mid-stance of a stride will probably be important, and the characteristic posture so derived may therefore bear considerable resemblance to it. 
797 Secondly, many assumptions and modelling simplifications were necessarily made in this study.

798 Many of these were necessitated by the lack of empirical data for extinct, non-avian theropods, 799 such as soft tissue anatomy or material properties, which in all likelihood will never be obtained.

800 Other simplifications pertained to making the system more tractable for analysis and 801 interpretation, such as representing the knee and ankle joints with a single degree of freedom 802 each, when it is known that these joints are capable of more complex motions during locomotion 803 in birds (Kambic et al. 2014; Rubenson et al. 2007). All of the assumptions and simplifications 804 involved in the present study could in principle be investigated via sensitivity analysis, but no 805 such analysis was performed here, save for the assumption of constant maximum force across all 806 musculotendon actuators (see next section below). All other assumptions were kept at their 'best 807 guess' manifestation throughout the study. By keeping every aspect of every stage of the 808 modelling process constant, and only varying posture, this allowed for the direct comparison of 809 simulation results to postural differences: differences in model results were entirely due to 810 differences in limb posture. When these assumptions are also held constant in a comparative 811 context across species (Bright 2014), this also allows for a more direct assessment of the effects 812 of posture on limb bone loading and muscular recruitment (Part III).

\section{II.3.8 Sensitivity to muscle forces}

\section{6}

817 In the musculoskeletal simulations, all musculotendon actuators were assigned the same 818 maximum force (2 BW) for the sake of simplicity and also to facilitate consistency across extinct 819 and extant theropod species. In reality, the varying sizes and architectures of the different muscles mean that they can have greatly different maximal force capabilities, which may have an important effect on the end results. To examine how sensitive the results were to using more realistic muscle force capabilities, the solution posture identified above was re-analysed with muscle-specific maximum force capacities stipulated. The original chicken carcass used to build the model was not able to be dissected for measurement of muscle architecture, and so the data collected by Paxton et al. (2010) for an adult $(2.08 \mathrm{~kg})$ junglefowl were used instead, scaled to the chicken model in proportion to mass. Maximum muscle force for each of the active muscles 
827 was then calculated following standard formulae, assuming a constant isometric stress of $3 \times 10^{5}$

$828 \mathrm{~N} / \mathrm{m}^{2}$ (Medler 2002); values are reported in Table 4. The maximum force for the four collateral

829 ligaments modelled was left unaltered from the original values.

830

831 The results for both the musculoskeletal and finite element simulations of this sensitivity test are

832 reported below alongside those for the main simulations.

833

834

835

\section{II.4 Results}

836

837

A total of eight different postures were tested before no closer correspondence between principal stress trajectories and cancellous bone architectural patterns was achieved. This was determined from both the qualitative visual inspection of stress trajectories and architectural patterns in all three bones, as well as quantitative minimization of angular deviation between stresses and fabric directions in the femoral head and medial femoral condyle. Going from the worst to best postures tested, the angular deviation between the minimum principal stress $\left(\boldsymbol{\sigma}_{3}\right)$ and the primary fabric orientation $\left(\mathbf{u}_{1}\right)$ in the femoral head decreased from $23.3^{\circ}$ to $7.9^{\circ}$, a $66 \%$ reduction; likewise, the angular deviation between $\boldsymbol{\sigma}_{3}$ and $\mathbf{u}_{1}$ in the medial femoral condyle decreased from $29.2^{\circ}$ to $17.3^{\circ}$, a $41 \%$ reduction. The final solution posture is illustrated in the centre of Fig. 4. The posture, as empirically predicted from the total leg length of the chicken individual modelled

848 (275 mm), would be 0.166 (Bishop et al. 2018). It is worth remembering that despite this close 849 similarity, the solution posture should not be equated literally with any single real posture used 850 (be it of standing, slow walking, fast running, etc.), for it is a characteristic weighted average of 851 all postures used.

852

853

854

855

856

857

\section{II.4.1 Principal stress trajectories}


859 In the solution posture, the principal stress trajectories in the femur, in particular those of $\boldsymbol{\sigma}_{3}$

860 (compressive), showed a high degree of correspondence with the observed cancellous bone

861 architectural directions, in the femoral head, under the facies antitrochanterica, in the trochanteric

862 crest and in both femoral condyles (Fig. 5A-P). The mean direction of $\sigma_{3}$ in the femoral head

863 showed strong correspondence to the mean direction of $\mathbf{u}_{1}$ measured for birds (Fig. 5Q);

864 compared to just the mean direction for the chickens studied in Part I $(n=2)$, the mean direction

865 of $\sigma_{3}$ had the same general azimuth, but was less proximally inclined (angular deviation of $18^{\circ}$ ).

866 Fair correspondence between $\boldsymbol{\sigma}_{3}$ and $\mathbf{u}_{1}$ also occurred in the medial femoral condyle, although the

867 direction of $\boldsymbol{\sigma}_{3}$ was notably more posteriorly inclined than the mean direction of $\mathbf{u}_{1}$ across all

868 birds (Fig. 5R); a more posteriorly inclined orientation of $\sigma_{3}$ occurred in all postures tested.

869

870

Much correspondence between principal stress trajectories and cancellous bone architecture was also observed in the tibiotarsus, particularly in the proximal end (Fig. 6). In the anterior cnemial crest, the trajectory of the maximum principal stress $\left(\boldsymbol{\sigma}_{1}\right.$, tensile) largely paralleled the margins of the crest, as observed for cancellous bone fabric. In much of the lateral cnemial crest, the observed cancellous bone fabric reported for birds was reflected by the trajectory of $\boldsymbol{\sigma}_{3}$. Under the articular facies, the trajectory of $\boldsymbol{\sigma}_{3}$ corresponded closely with the observed architectural patterns there, showing a posterior inclination largely parallel to the sagittal plane. Additionally, in the sagittal plane through the middle of the proximal end, $\boldsymbol{\sigma}_{1}$ and $\boldsymbol{\sigma}_{3}$ formed a double-arcuate pattern, closely resembling a similar pattern in $\mathbf{u}_{1}$ observed in some of the large bird individuals studied in Part I (Fig. 6S,T). In contrast to the proximal tibiotarsus, only minimal correspondence between principal stress trajectories and cancellous bone architecture could be attained in the distal tibiotarsus, in any posture tested. In the solution posture, there was some correspondence between $\boldsymbol{\sigma}_{3}$ and observed architecture in the immediate vicinity of the articular condyles, where $\boldsymbol{\sigma}_{3}$ was largely parallel to the sagittal plane (Fig. $6 \mathrm{U}, \mathrm{V}$ ), but this was not observed throughout the entire distal end of the bone, unlike the architecture. inclination observed in the cancellous bone architecture (Fig. 7). Medially, $\boldsymbol{\sigma}_{1}$ showed this 


\section{II.4.2 Mid-shaft stresses}

892

893

In the solution posture, the most axis-parallel orientation of both $\boldsymbol{\sigma}_{1}$ and $\boldsymbol{\sigma}_{3}$ at the femoral mid894 shaft was at a high angle to the long-axis of the bone, by at least $30^{\circ}$, indicating considerable torsion (Fig. 8A). Moreover, the sense of torsion as indicated by the stress trajectories was positive; when the right femur is viewed proximally, the proximal end was rotated counterclockwise relative to the distal end (Fig. 8B). The neutral axis of bending was oriented $36^{\circ}$ from the mediolateral axis, indicating that bending of the femur was predominantly in an anteroposterior direction.

900

901

In the tibial mid-shaft, the most axis-parallel orientation of $\boldsymbol{\sigma}_{1}$ and $\boldsymbol{\sigma}_{3}$ was almost parallel to the long-axis of the bone, indicating only a minimal torsion (Fig. 8C). The sense of torsion (what very little there is) as indicated by the stress trajectories was also positive. The neutral axis of bending was oriented $19^{\circ}$ from the mediolateral axis, indicating that bending of the tibiotarsus was also in a predominantly anteroposterior direction.

\section{II.4.3 Muscle and ligament activations}

909

910

In the solution posture, the activations of the four collateral ligament actuators were very low

911 (0.012 or less), indicating that the vast majority of limb stabilization, excluding the

912 metatarsophalangeal joint, was conferred by muscle actuators. However, as the knee and ankle were represented as hinge joints in this study, joint stabilization would also have been achieved in

914 part through resistance offered by these single degree-of-freedom joints to off-axis moments and

915 forces. (Indeed, this resistance could well be responsible for the minimal recruitment of ligaments

916 in the first place.) This resistance was nevertheless transmitted to the bones as joint moments and

917 forces (calculated in the musculoskeletal simulations). Therefore, as far as the bones are

918 concerned, all experienced loads are accounted for and incorporated into the finite element 919 simulations. Nevertheless, as off-axis loads were able to be partly resisted by the hinge joints, the 
920 calculated forces in the collateral ligaments may be appreciably less than what they would be in

921 vivo.

922

923 The activations of all muscle actuators are presented in Fig. 8D. Most muscles were recruited

924 with activations above 0.1 (i.e., clearly active); no muscle was recruited beyond half of its

925 maximal capacity. The iliotrochanterici medius (ITM) et caudalis (ITC), grastrocnemius medialis

926 (GM) and fibularis longus (FL) were the most recruited muscles, each with an activation above

927 0.4. The iliofibularis (ILFB), ischiofemoralis (ISF) and caudofemorales partes caudalis (CFC) et

928 pelvica (CFP) were the least recruited muscles, all with negligible activations.

929

930

931

\section{II.4.4 Muscle force sensitivity test}

932

933 The activations of muscle actuators in the sensitivity test were sometimes markedly different 934 from those of the original simulation (Fig. 8D). However, there was also agreement between the 935 two simulations, where several key hindlimb muscles were predicted to be important in both 936 cases (e.g., ITC, FMTI, GM, GL and FL). Despite the differences in muscle maximum force 937 capabilities between the original and sensitivity test simulations (Table 4), as well as the 938 differences in calculated muscle activations, the stress results differed little from those of the 939 original simulation. The qualitative patterns of stress trajectories were highly similar to those of

940 the original simulation in most regions of the femur, tibiotarsus and fibula. The only region where 941 there was a marked difference was in part of the cranial cnemial crest, in terms of $\sigma_{1}$ (Fig. 6E, F, 942 cf. Fig. 6A,C). In terms of quantitative results, there was again little deviation in the mean 943 direction of $\boldsymbol{\sigma}_{3}$ from that calculated for the original simulation (Fig. 5Q, R, orange dots). The 944 difference in mean $\boldsymbol{\sigma}_{3}$ directions between the original and sensitivity test simulations was very 945 small in both the femoral head $\left(1.3^{\circ}\right)$ and medial femoral condyle $\left(5.6^{\circ}\right)$. These results suggest 946 that the approach of assigning a single constant value of $2 \mathrm{BW}$ for muscle maximum force 947 capacity does not introduce a significant degree of error, at least as far as the objectives of the 948 present study are concerned. 
$951 \underline{\text { II.5 Discussion }}$

952

953 The aim of this study was to verify the 'reverse' application of the trajectorial theory, to go from

954 observed cancellous bone architectural patterns to bone loading regimes and limb postures, as

955 applied to theropod locomotor biomechanics. This was achieved through the development of a

956 novel approach that integrated musculoskeletal and finite element simulations of a modern

957 theropod, the chicken. By focusing on a modern theropod here, the validity of the reverse

958 approach was able to examined for each major bone in the hindlimb.

959

960

961

\section{II.5.1 Successes and pitfalls}

962

963

Despite the many modelling simplifications made in the current study, and that only a single

964

static posture was modelled for any one test, much of the observed cancellous bone architectural

965 patterns in the avian hindlimb was able to be replicated in the principal stress trajectories. This was particularly true of the femur. The 'solution posture' that produced the greatest correspondence between principal stresses and cancellous bone architecture is qualitatively comparable to the hindlimb posture of medium-sized birds such as chickens and guineafowl (Carrano \& Biewener 1999; Gatesy 1999a; Grossi et al. 2014), and less comparable to the postures of larger birds with more extended joints (Abourachid \& Renous 2000; Rubenson et al. 2007) or smaller birds with more flexed joints (Reilly 2000; Stoessel \& Fischer 2012).

972 Furthermore, the degree of crouch in the chicken model was almost identical to what would be empirically predicted based on limb bone lengths, for a quiet standing posture (Bishop et al. 2018). It is important to reiterate that the solution posture obtained here is a weighted average (by both time and load) of all postures and loading regimes experienced on a daily basis, and yet the relative weightings of each posture and loading regime are not actually known. Hence, it would

977 be worthwhile in future studies to more exactly discern what aspects of locomotor biomechanics 978 the solution posture most strongly reflects.

979

980

Other aspects of the solution posture also showed correspondence with empirical data for avian terrestrial locomotion. The femur was predicted to be loaded in considerable torsion, with a 
982 positive sense, as well as bending that was predominantly anteroposteriorly directed. This is

983 consistent with the loading regimes recorded by in vivo strain gauge studies of chickens and emus 984 (Carrano 1998; Carrano \& Biewener 1999; Main \& Biewener 2007). Additionally, the two most 985 strongly recruited muscles in the musculoskeletal simulations, the gastrocnemius medialis and 986 fibularis longus, are also the two largest muscles in the distal hindlimb of birds (Lamas et al. 987 2014; Paxton et al. 2010; Smith et al. 2006), and would therefore be expected to be capable of 988 producing large amounts of force, all other factors being equal.

989

990 There were also a few aspects in which the solution posture did not accord well with empirical 991 observations. Most pertinently, the principal stress trajectories in the distal tibiotarsus did not 992 show much correspondence with the cancellous bone architecture observed in this region of the 993 bone of birds (Part I). This may suggest that the manner in which that part of the bone was 994 modelled in the current study was inadequate, that is, too non-physiological. For instance, the 995 ankle joint moment calculated in the musculoskeletal simulations was not able to be applied in 996 the finite element simulations in their current formulation. The discrepancy may also be due to 997 the cancellous bone architecture of the distal tibiotarsus reflecting many different loading 998 regimes, any single one of which cannot capture the architecture. Some of those loading regimes 999 may be very different to that occurring around the mid-stance of locomotion, such as those 1000 associated with the swing phase of locomotion, or even standing and sitting. Alternatively, the 1001 poor correspondence may indicate that the trajectorial theory may not actually hold true here for 1002 some reason, potentially related to the ontogenetic fusion of the proximal tarsals and distal tibia 1003 of birds (see Lovejoy 2004; Lovejoy et al. 2002). A second aspect in which the solution posture 1004 did not concur with empirical observations concerned the stresses at the tibial mid-shaft. Here, 1005 the bone was predicted to be loaded in only minimal torsion, the sense of which was positive; this 1006 does not accord with in vivo strain gauge studies, which have shown that the avian tibiotarsus 1007 experiences a large amount of torsional loading during locomotion, which furthermore is of a 1008 negative sense (Biewener et al. 1986; Main \& Biewener 2007; Verner et al. 2016). It is possible 1009 that if additional degrees of freedom were assigned to the model (e.g., long-axis rotation in the 1010 knee or ankle; Kambic et al. 2014), more realistic results may have been achieved here (see also 1011 Bell et al. 2009, in relation to ornithischian dinosaur jaws). 
1013 A final incongruence between the solution posture and empirical observations was the negligible 1014 recruitment of some muscles in the static optimization routine of the musculoskeletal simulations. 1015 There were four such muscles (iliofibularis, ischiofemoralis and caudofemorales partes caudalis 1016 et pelvica), yet electromyography data indicates that at least three of these (iliofibularis, 1017 ischiofemoralis and caudofemoralis pars caudalis) are active during a significant part of the 1018 stance phase (Gatesy 1990; Gatesy 1999b; Jacobson \& Hollyday 1982; Marsh et al. 2004). The 1019 negligible recruitment of the ischiofemoralis and caudofemorales is consonant with the generally 1020 small size of these muscles in birds, but this does not hold for the iliofibularis, which is quite 1021 large (Hudson et al. 1959; Lamas et al. 2014; McGowan 1979; Patak \& Baldwin 1998; Paxton et al. 2010; Smith et al. 2006). It is probable that all four muscles were minimally recruited in the static optimization routine on account of (i) all muscle actuators were assigned the same maximum capable force output, and (ii) these four particular muscles have smaller moment arms of hip extension compared to other muscles, such as the flexores crures medialis, lateralis pars pelvica et lateralis pars accessoria (Fig. 8E). That is, the static optimization preferentially recruited muscles with larger moment arms, such that lower forces, and therefore activations, were required to provide the necessary stabilizing joint moments.

The last two aspects of discrepancy between the solution posture and empirical data may also in part reflect the fact that the musculoskeletal models were analysed as quasi-static systems. Dynamic aspects of locomotion, such as inertial forces or relative movement between bones, may lead to increased levels of torsion in the tibiotarsus. The same dynamic effects can also influence the net joint moments required to be stabilized by muscle forces; for instance, active retraction of the hip and flexion of the knee may lead to greater activation of the iliofibularis. Therefore, the activations calculated in the current study are probably most informative for muscles that predominantly confer postural stability, rather than active limb movement (i.e., those that act as brakes or motors; Rankin et al. 2016). 
1043 Numerous assumptions and modelling simplifications were made in the course of this study, and 1044 these have already been discussed in detail above. However, this study had other attendant 1045 limitations, which are noted here and which provide the basis for future work. Owing to the 1046 constraints of time and resources, it was only feasible to model a single avian species to test the 1047 validity of the reverse application of the trajectorial theory. In the context of the present study's 1048 objectives, this was deemed sufficient; yet, the modelling of additional species in the future 1049 (across the size spectrum of extant birds) could help to further clarify the strengths and 1050 weaknesses of the approach. A further limitation of this study concerns the use of quantitative 1051 comparisons of principal stresses and cancellous bone fabric, which was restricted to two regions of the femur. This was in part due to time constraints, but it also stemmed from similarly restricted quantification of cancellous bone fabric in Part I of this series. It will be informative in future work to expand the number of regions of the femur subjected to quantitative comparisons, and expand this to include the tibiotarsus and fibula as well. Such an expanded quantification will likely have to negotiate region-specific obstacles to consistent calculation, such as the anteroposterior 'fanning' of fabric vectors in the distal femoral condyles (Part I). Not only will expanded quantification improve the rigour of analyses and comparisons, but it may also lend itself to the implementation of a more formal (and automated) optimization approach to deriving a solution posture for a given species. Furthermore, the use of a more automated approach may allow additional degrees of freedom to be tractably incorporated into the models (e.g., in the

Notwithstanding the aforementioned discrepancies and limitations, the concept of applying the trajectorial theory in reverse is, overall, well-supported by the results of the present study. The reverse approach therefore has the potential to provide insight into the loading regimes experienced by extinct, non-avian theropods during locomotion, and more broadly the postures used during locomotion. 
1073 Previous studies have sought to use the architecture of cancellous bone to derive loading 1074 conditions experienced in vivo, although this has largely been confined to theoretical studies of 1075 modern animals. Some of these studies have focused on utilizing the spatial distribution of the 1076 bulk density of cancellous bone, to which remodelling algorithms (Bona et al. 2006; Fischer et al. 1077 1995) or artificial neural networks (Campoli et al. 2012; Zadpoor et al. 2013) are applied to 1078 retrieve one or more loading regimes. Presently, these studies have only been implemented in two 1079 dimensions, and so their efficacy in analysing complex, 3-D geometries or loading regimes (such 1080 as torsion) remains to be determined. More importantly for the study of extinct species, however, 1081 the process of fossilization will greatly hamper any attempt founded upon the bulk density of 1082 cancellous bone. Geological chemical alteration (diagenesis) has the potential to greatly alter the 1083 physical density of a fossil bone, and moreover this alteration may not be uniform across a bone, 1084 1085 such that it may be impossible to reconstruct the original patterns of bulk density in the living bone.

1086

1087

Diagenesis does not, however, normally alter the actual structure of bone; indeed, fine, cellular1088 level structures are frequently preserved in the fossil bones of dinosaurs and other vertebrates (Chinsamy-Turan 2005; Houssaye 2014). It is the structural characteristics of cancellous bone architecture that are utilized in the present study, namely, fabric directions. Regardless of alterations to bulk density, so long as the actual structure of cancellous bone is preserved in a given fossil, and can be imaged appropriately, then the approach of the present study is feasible. (Of course, bones or regions thereof that have suffered taphonomic deformation should be avoided; Bishop et al. 2017a.) The structural characteristics of cancellous bone have also been used previously in the deduction of in vivo loads, in a series of studies by Christen et al. (2012, 2013a,b, 2015). These studies developed voxel-based micro-finite element models that modelled each individual trabecula of a bone, and sought to determine the loading regime, or combination of loading regimes, that achieved the most uniform distribution of strain energy density across the model. The great geometric complexity of the models used in these studies necessitated immense computational capability; only small bones or parts of larger ones were able to be modelled. The computational requirements would quickly become prohibitively large for the modelling of whole bones of even a modest size. Moreover, such geometric complexity would be impossible to produce for medium-sized or large bones, for which high-resolution CT imagery is currently 
1104 unattainable. An additional problem faced by these computational studies is that currently only

1105 very basic loads are able to be examined, and these are only applied at the joints; muscle forces

1106 were not considered.

1107

1108 In light of the above discussion, the advantages of the reverse trajectorial approach developed in

1109 the current study are clear. Firstly, it is based on the actual structure of cancellous bone, which is

1110 usually resistant to alteration by diagenetic processes. Moreover, the structural information

1111 required (i.e., fabric directionality) can be ascertained for specimens of a wide range of sizes;

1112 each individual trabecula need not be imaged in micron-level resolution, although how the image

1113 data is acquired and analysed may vary with the size of the specimen, as demonstrated in Part I.

1114 The reverse approach is also easily implemented as a fully 3-D analysis that is relatively

1115 computationally inexpensive to perform; using a computer with $32 \mathrm{~Gb}$ of memory and a $2.4 \mathrm{GHz}$

1116 processor, no single simulation of the present study took more than 10 minutes to solve.

1117 However, the main advantage of the reverse approach is that it explicitly links whole-bone

1118 cancellous architecture to whole-limb musculoskeletal mechanics. Thus, cancellous bone

1119 architectural patterns can be used to directly test hypotheses of limb posture, muscle control and

1120 bone loading mechanics, as will be done in Part III.

\section{$\underline{\text { II.6 Conclusion }}$}

In this study a new, mechanistic approach to reconstructing locomotor biomechanics in theropods was developed and tested. Its underlying concept of applying the trajectorial theory in reverse was overall well-supported by the results of the present study: cancellous bone architecture can be used to derive bone loading regimes, and in turn limb postures. This is achieved through the integration of 3-D musculoskeletal and finite element models with observations of cancellous bone fabric direction.

With just a single, quasi-static posture of a chicken hindlimb, modelled with a number of relatively simple assumptions, a large portion of the observed patterns in cancellous bone architecture in birds was able to be replicated by principal stress trajectories. This posture 
1135

1136

1137

1138

1139

1140

1141

1142

1143

1144

1145

1146

1147

1148

1149

1150

1151

1152

1153

1154

1155

1156

1157

1158

1159

1160

1161

1162

1163

1164

1165

correlated to those actually used during locomotion in birds, in particular the postures used at around mid-stance of normal terrestrial locomotion. Additionally, other biomechanical aspects of the posture, including loading mechanics of the femur and the activations of certain muscles, corresponded well to empirical data recorded for birds. Less agreement between principal stresses and cancellous bone fabric was achievable in the distal tibiotarsus, which requires further investigation, possibly involving more complex modelling approaches (e.g., incorporating more degrees of freedom, or incorporating dynamic effects such as segment accelerations and relative movement between bones).

The reverse approach therefore holds great promise for better understanding whole-bone and whole-limb musculoskeletal biomechanics in the hindlimbs of non-avian theropods during terrestrial locomotion. The generality of this approach also means that it could also be used to improve understanding of locomotor biomechanics in other extinct tetrapod vertebrate groups as well. As correspondence between principal stresses and cancellous bone architecture was greatest in the femur in the present study, this suggests that the reverse approach will yield the most insight for more proximal limb segments.

\section{II.7 Acknowledgements}

The staff of the Geosciences Program of the Queensland Museum is thanked for the provision of workspace and access to literature: A. Rozefelds, K. Spring, R. Lawrence, P. Tierney, J. Wilkinson and D. Lewis. Much appreciation is extended to N. Newman (Queensland X-ray, Brisbane) and R. Lawrence for assistance with CT scanning. The thorough and constructive comments on earlier versions of the manuscript, provided by S. Gatesy, T. Ryan, D. Henderson, E. Snively and an anonymous reviewer, are all greatly appreciated, and substantially improved the clarity and content of the research presented here. All scripts and data used are held in the Geosciences Collection of the Queensland Museum, and will be made available upon request to the Collections Manager. 
1166

1167

1168

1169

1170

1171

1172

1173

1174

1175

1176

1177

1178

1179

1180

1181

1182

1183

1184

1185

1186

1187

1188

1189

1190

1191

1192

1193

1194

1195

1196

1197

1198

1199

1200

1201

1202

1203

1204

1205

1206

1207

1208

1209

1210

1211

\section{II.8 References}

Abourachid A, Hackert R, Herbin M, Libourel PA, Lambert F, Gioanni H, Provini P, Blazevic P, and Hugel V. 2011. Bird terrestrial locomotion as revealed by 3-D kinematics. Zoology 114:360-368.

Abourachid A, and Renous S. 2000. Bipedal locomotion in ratites (Paleognatiform): examples of cursorial birds. Ibis 142:538-549.

Adachi T, Tsubota K, Tomita Y, and Hollister SJ. 2001. Trabecular Surface Remodeling Simulation for Cancellous Bone Using Microstructural Voxel Finite Element Models. Journal of Biomechanical Engineering 123:403-409.

Alexander RM. 1977. Terrestrial locomotion. In: Alexander RM, and Goldspink G, eds. Mechanics and Energetics of Animal Locomotion. London: Chapman and Hall, 168-203.

Allen V, Bates KT, Li Z, and Hutchinson JR. 2013. Linking the evolution of body shape and locomotor biomechanics in bird-line archosaurs. Nature 497:104-107.

Allen V, Kambic RE, Gatesy SM, and Hutchinson JR. 2017. Gearing effects of the patella (knee extensor muscle sesamoid) of the helmeted guineafowl during terrestrial locomotion. Journal of Zoology 303:178-187.

Allmendinger RW, Cardozo NC, and Fisher D. 2013. Structural Geology Algorithms: Vectors and Tensors. Cambridge: Cambridge University Press.

Amson E, Arnold P, van Heteren AH, Canoville A, and Nyakatura JA. 2017. Trabecular architecture in the forelimb epiphyses of extant xenarthrans (Mammalia). Frontiers in Zoology 14:52.

Anderson FC, and Pandy MG. 1999. A Dynamic Optimization Solution for Vertical Jumping in Three Dimensions. Computer Methods in Biomechanics and Biomedical Engineering 2:201-231.

Anderson FC, and Pandy MG. 2001. Static and dynamic optimization solutions for gait are practically equivalent. Journal of Biomechanics 34:153-161.

Andrada E, Nyakatura JA, Bergmann F, and Blickhan R. 2013. Adjustments of global and local hindlimb properties during terrestrial locomotion of the common quail (Coturnix coturnix). Journal of Experimental Biology 216:3906-3916.

Attene M, and Falcidieno B. 2006. ReMESH: An Interactive Environment to Edit and Repair Triangle Meshes. Proceedings of the Eighth International Conference on Shape Modeling and Applications. Matushima. p 271-276.

Barak MM, Lieberman DE, and Hublin J-J. 2011. A Wolff in sheep's clothing: Trabecular bone adaptation in response to changes in joint loading orientation. Bone 49:1141-1151.

Barak MM, Lieberman DE, Raichlen DA, Pontzer H, Warrener AG, and Hublin J-J. 2013. Trabecular Evidence for a Human-Like Gait in Australopithecus africanus. PLoS ONE 8:e77687.

Beaupré GS, Orr TE, and Carter DR. 1990. An Approach for Time-Dependent Bone Modeling and Remodeling - Application: A Preliminary Remodeling Simulation. Journal of Orthopaedic Research 8:662-670.

Beer FP, Johnston ER, Jr, DeWolf JT, and Mazurek DF. 2012. Mechanics of Materials. New York: McGraw-Hill.

Bell PR, Snively E, and Shychoski L. 2009. A Comparison of the Jaw Mechanics in Hadrosaurid and Ceratopsid Dinosaurs Using Finite Element Analysis. The Anatomical Record 292:1338-1351. 
1212 Bergmann G, Deuretzbacher G, Heller M, Graichen F, Rohlmann A, Strauss J, and Duda GN.

1213

1214

1215

1216

1217

1218

1219

1220

1221

1222

1223

1224

1225

1226

1227

1228

1229

1230

1231

1232

1233

1234

1235

1236

1237

1238

1239

1240

1241

1242

1243

1244

1245

1246

1247

1248

1249

1250

1251

1252

1253

1254

1255

1256
2001. Hip contact forces and gait patterns from routine activities. Journal of Biomechanics 34:859-871.

Bergmann G, Graichen F, and Rohlmann A. 1999. Hip joint forces in sheep. Journal of Biomechanics 32:769-777.

Biewener AA, Fazzalari NL, Konieczynski DD, and Baudinette RV. 1996. Adaptive Changes in Trabecular Architecture in Relation to Functional Strain Patterns and Disuse. Bone 19:18.

Biewener AA, Swartz SM, and Bertram JEA. 1986. Bone Modelling During Growth: Dynamics Strain Equilibrium in the Chick Tibiotarsus. Calcified Tissue International 39:390-395.

Bishop PJ, Clemente CJ, Graham DF, Lamas LP, Hutchinson JR, Rubenson J, Hancock JA, Wilson RS, Hocknull SA, Barrett RS, and Lloyd DG. 2018. The Influence of Speed and Size on Avian Terrestrial Locomotor Biomechanics: Predicting Locomotion in Extinct Theropod Dinosaurs. PLoS ONE 13:e192172.

Bishop PJ, Clemente CJ, Hocknull SA, Barrett RS, and Lloyd DG. 2017a. The effects of cracks on the quantification of the cancellous bone fabric tensor in fossil and archaeological specimens: a simulation study. Journal of Anatomy 230:461-470.

Bishop PJ, Clemente CJ, Weems RE, Graham DF, Lamas LP, Hutchinson JR, Rubenson J, Wilson RS, Hocknull SA, Barrett RS, and Lloyd DG. 2017b. Using step width to compare locomotor biomechanics between extinct, non-avian theropod dinosaurs and modern obligate bipeds. Journal of the Royal Society Interface 14:20170276.

Bishop PJ, Hocknull SA, Clemente CJ, Hutchinson JR, Farke AA, Barrett RS, and Lloyd DG. in review-a. Cancellous bone architecture and theropod dinosaur locomotion. Part III Inferring posture and locomotor biomechanics in extinct theropods, and its evolution on the line to birds. PeerJ.

Bishop PJ, Hocknull SA, Clemente CJ, Hutchinson JR, Farke AA, Beck BR, Barrett RS, and Lloyd DG. in review-b. Cancellous bone architecture and theropod dinosaur locomotion. Part I - An examination of cancellous bone architecture in the hindlimb bones of theropods. PeerJ.

Blob RW, and Biewener AA. 2001. Mechanics of limb bone loading during terrestrial locomotion in the green iguana (Iguana iguana) and American alligator (Alligator mississippiensis). Journal of Experimental Biology 204:1099-1122.

Bona MA, Martin LD, and Fischer KJ. 2006. A contact algorithm for density-based load estimation. Journal of Biomechanics 39:636-644.

Bonnan MF, Sandrik JL, Nishiwaki T, Wilhite DR, Elsey RM, and Vittore C. 2010. Calcified Cartilage Shape in Archosaur Long Bones Reflects Overlying Joint Shape in StressBearing Elements: Implications for Nonavian Dinosaur Locomotion. The Anatomical Record 293:2044-2055.

Bonnan MF, Wilhite DR, Masters SL, Yates AM, Gardner CK, and Aguiar A. 2013. What Lies Beneath: Sub-Articular Long Bone Shape Scaling in Eutherian Mammals and Saurischian Dinosaurs Suggests Different Locomotor Adaptations for Gigantism. PLoS ONE 8:e75216.

Boyle C, and Kim IY. 2011. Three-dimensional micro-level computational study of Wolff's law via trabecular bone remodeling in the human proximal femur using design space topology optimization. Journal of Biomechanics 44:935-942. 
1257

1258

1259

1260

1261

1262

1263

1264

1265

1266

1267

1268

1269

1270

1271

1272

1273

1274

1275

1276

1277

1278

1279

1280

1281

1282

1283

1284

1285

1286

1287

1288

1289

1290

1291

1292

1293

1294

1295

1296

1297

1298

1299

1300

1301

1302

Brassey CA, Maidment SCR, and Barrett PM. 2017. Muscle moment arm analyses applied to vertebrate paleontology: a case study using Stegosaurus stenops Marsh, 1887. Journal of Vertebrate Paleontology 37:e1361432.

Bright JA. 2014. A review of paleontological finite element models and their validity. Journal of Paleontology 88:760-769.

Bright JA, and Rayfield EJ. 2011. The Response of Cranial Biomechanical Finite Element Models to Variations in Mesh Density. The Anatomical Record 294:610-620.

Bryant JD, Bennett MB, Brust J, and Alexander RM. 1987. Forces exerted on the ground by galloping dogs (Canis familiaris). Journal of Zoology 213:193-203.

Butcher MT, and Blob RW. 2008. Mechanics of limb bone loading during terrestrial locomotion in river cooter turtles (Pseudemys concinna). Journal of Experimental Biology 211:11871202.

Calow LJ, and Alexander RM. 1973. A mechanical analysis of a hind leg of a frog (Rana temporaria). Journal of Zoology 171:293-321.

Campoli G, Weinans H, and Zadpoor AA. 2012. Computational load estimation of the femur. Journal of the Mechanical Behaviour of Biomedical Materials 10:108-119.

Cardozo NC, and Allmendinger RW. 2013. Spherical projections with OSXStereonet. Computers \& Geosciences 51:193-205.

Carey RE, Zheng L, Aiyangar AK, Harner CD, and Zhang X. 2014. Subject-Specific Finite Element Modeling of the Tibiofemoral Joint Based on CT, Magnetic Resonance Imaging and Dynamic Stereo-Radiography Data in Vivo. Journal of Biomechanical Engineering 136:041004-041001.

Carrano MT. 1998. Locomotion in non-avian dinosaurs: integrating data from hindlimb kinematics, in vivo strains, and bone morphology. Paleobiology 24:450-469.

Carrano MT, and Biewener AA. 1999. Experimental Alteration of Limb Posture in the Chicken (Gallus gallus) and its Bearing on the Use of Birds as Analogs for Dinosaur Locomotion. Journal of Morphology 240:237-249.

Carrano MT, and Hutchinson JR. 2002. Pelvic and hindlimb musculature of Tyrannosaurus rex (Dinosauria: Theropoda). Journal of Morphology 253:207-228.

Carter DR, and Beaupré GS. 2001. Skeletal Function and Form: Mechanobiology of Skeletal Development, Aging, and Regeneration. Cambridge: Cambridge University Press.

Carter DR, Orr TE, and Fyhrie DP. 1989. Relationships between loading history and femoral cancellous bone architecture. Journal of Biomechanics 22:231-244.

Chadwick KP, Regnault S, Allen V, and Hutchinson JR. 2014. Three-dimensional anatomy of the ostrich (Struthio camelus) knee joint. PeerJ 2:e706.

Charles JP, Cappellari O, Spence AJ, Hutchinson JR, and Wells DJ. 2016. Musculoskeletal Geometry, Muscle Architecture and Functional Specialisations of the Mouse Hindlimb. PLoS ONE 11:e147669.

Chinsamy-Turan A. 2005. The Microstructure of Dinosaur Bone. Baltimore: Johns Hopkins University Press.

Christen P, Ito K, dos Santos AA, Müller R, and van Rietbergen B. 2013a. Validation of a bone loading estimation algorithm for patient-specific bone remodelling simulations. Journal of Biomechanics 46:941-948.

Christen P, Ito K, Galis F, and van Rietbergen B. 2015. Determination of hip-joint loading patterns of living and extinct mammals using an inverse Wolff's law approach. Biomechanics and Modeling in Mechanobiology 14:427-432. 
1303 Christen P, Ito K, Knippels I, Müller R, van Lenthe GH, and van Rietbergen B. 2013b. Subject-

1304

1305

1306

1307

1308

1309

1310

1311

1312

1313

1314

1315

1316

1317

1318

1319

1320

1321

1322

1323

1324

1325

1326

1327

1328

1329

1330

1331

1332

1333

1334

1335

1336

1337

1338

1339

1340

1341

1342

1343

1344

1345

1346

1347

1348 specific bone loading estimation in the human distal radius. Journal of Biomechanics 46:759-766.

Christen P, van Rietbergen B, Lambers FM, Müller R, and Ito K. 2012. Bone morphology allows estimation of loading history in a murine model of bone adaptation. Biomechanics and Modeling in Mechanobiology 11:483-492.

Coelho PG, Fernandes PR, Rodrigues HC, Cardoso JB, and Guedes JM. 2009. Numerical modeling of bone tissue adaptation-A hierarchical approach for bone apparent density and trabecular structure. Journal of Biomechanics 42:830-837.

Cowin SC. 2001. The false premise in Wolff's Law. In: Cowin SC, ed. Bone Biomechanics Handbook. 2 ed. Boca Raton: CRC Press, 30-31-30-15.

Currey JD. 2002. Bones: Structure and Mechanics. Princeton: Princeton University Press.

Delp SL, Anderson FC, Arnold AS, Loan P, Habib A, John CT, Guendelman E, and Thelen DG. 2007. OpenSim: Open-Source Software to Create and Analyze Dynamic Simulations of Movement. IEEE Transactions of Biomedical Engineering 54:1940-1950.

Delp SL, Loan P, Hoy MG, Zajac FE, Topp EL, and Rosen JM. 1990. An Interactive GraphicsBased Model of the Lower Extremity to Study Orthopaedic Surgical Procedures. IEEE Transactions of Biomedical Engineering 37:757-767.

Dumont ER, Piccirillo J, and Grosse IR. 2005. Finite-Element Analysis of Biting Behaviour and Bone Stress in the Facial Skeletons of Bats. The Anatomical Record 283A:319-330.

Erickson GM, Catanese J, III, and Keaveny TM. 2002. Evolution of the Biomechanical Properties of the Femur. The Anatomical Record 268:115-124.

Fajardo RJ, and Müller R. 2001. Three-Dimensional Analysis of Nonhuman Primate Trabecular Architecture Using Micro-Computed Tomography. American Journal of Physical Anthropology 115:327-336.

Fernandez JW, Mithraratne P, Thrupp SF, Tawhai MH, and Hunter PJ. 2004. Anatomically based geometric modelling of the musculo-skeletal system and other organs. Biomechanics and Modeling in Mechanobiology 2:139-155.

Fischer KJ, Jacobs CR, and Carter DR. 1995. Computational method for determination of bone and joint loads using bone density distributions. Journal of Biomechanics 28:1127-1135.

Gatesy SM. 1990. Caudofemoral musculature and the evolution of theropod locomotion. Paleobiology 16:170-186.

Gatesy SM. 1994. Neuromuscular Diversity in Archosaur Deep Dorsal Thigh Muscles. Brain, Behavior and Evolution 43:1-14.

Gatesy SM. 1999a. Guineafowl Hindlimb Function I: Cineradiographic Analysis and Speed Effects. Journal of Morphology 240:115-125.

Gatesy SM. 1999b. Guineafowl Hindlimb Function II: Electromyographic Analysis and Motor Pattern Evolution. Journal of Morphology 240:127-142.

Gefen A, and Seliktar R. 2004. Comparison of the trabecular architecture and the isostatic stress flow in the human calcaneus. Medical Engineering \& Physics 26:119-129.

Geraldes DM, Modenese L, and Phillips ATM. 2016. Consideration of multiple load cases is critical in modelling orthotropic bone adaptation in the femur. Biomechanics and Modeling in Mechanobiology 15:1029-1042.

Geraldes DM, and Phillips ATM. 2014. A comparative study of orthotropic and isotropic bone adaptation in the femur. International Journal for Numerical Methods in Biomedical Engineering 30:873-889. 
1349

1350

1351

1352

1353

1354

1355

1356

1357

1358

1359

1360

1361

1362

1363

1364

1365

1366

1367

1368

1369

1370

1371

1372

1373

1374

1375

1376

1377

1378

1379

1380

1381

1382

1383

1384

1385

1386

1387

1388

1389

1390

1391

1392

1393

Giarmatzis G, Jonkers I, Wesseling M, Van Rossom S, and Verschueren S. 2015. Loading of Hip Measured by Hip Contact Forces at Different Speeds of Walking and Running. Journal of Bone and Mineral Research 30:1431-1440.

Giddings VL, Beaupré GS, Whalen RT, and Carter DR. 2000. Calcaneal loading during walking and running. Medicine \& Science in Sports \& Exercise 32:627-634.

Gilbert MM, Snively E, and Cotton J. 2016. The Tarsometatarsus of the Ostrich Struthio camelus: Anatomy, Bone Densities, and Structural Mechanics. PLoS ONE 11:e0149708.

Goetz JA, Derrick TR, Pedersen DR, Robinson DA, Conzemius MG, Baer TE, and Brown TD. 2008. Hip joint contact force in the emu (Dromaius novaehollandiae) during normal level walking. Journal of Biomechanics 41:770-778.

Goldstein SA, Matthews LS, Kuhn JL, and Hollister SJ. 1991. Trabecular bone remodelling: an experimental model. Journal of Biomechanics 24 (suppl. 1):135-150.

Gosnell WC, Butcher MT, Maie T, and Blob RW. 2011. Femoral loading mechanics in the Virginia opossum, Didelphis virginiana: torsion and mediolateral bending in mammalian locomotion. Journal of Experimental Biology 214:3455-3466.

Griffin NL, D’Août K, Ryan TM, Richmond BG, Ketcham RA, and Postnov A. 2010. Comparative forefoot trabecular bone architecture in extant hominids. Journal of Human Evolution 59:202-213.

Grossi B, Iriarte-Díaz J, Larach O, Canals M, and Vásquez RA. 2014. Walking Like Dinosaurs: Chickens with Artificial Tails Provide Clues about Non-Avian Theropod Locomotion. PLOS ONE 9:e88458.

Guldman RE, Richards M, Caldwell NJ, Kuelske CL, and Goldstein SA. 1997. Trabecular bone adaptation to variations in porous-coated implant topology. Journal of Biomechanics 30:147-153.

Haines RW. 1942. The tetrapod knee joint. Journal of Anatomy 76:270-301.

Hayes WC, and Snyder B. 1981. Toward a quantitative formulation of Wolff's Law in trabecular bone. In: Cowin SC, ed. Mechanical Properties of Bone. New York: American Society of Mechanical Engineers, 43-68.

Hébert D, Lebrun R, and Marivaux L. 2012. Comparative Three-Dimensional Structure of the Trabecular Bone in the Talus of Primates and Its Relationship to Ankle Joint Loads Generated During Locomotion. The Anatomical Record 295:2069-2088.

Houssaye A. 2014. Advances in vertebrate palaeohistology: recent progress, discoveries, and new approaches. Biological Journal of the Linnean Society 112:645-648.

Hudson GE. 1937. Studies on the Muscles of the Pelvic Appendage in Birds. American Midland Naturalist 18:1-108.

Hudson GE, Lanzillotti PJ, and Edwards GD. 1959. Muscles of the Pelvic Limb in Galliform Birds. American Midland Naturalist 61:1-67.

Huiskes R, Ruimerman R, van Lenthe GH, and Janssen JD. 2000. Effects of mechanical forces on maintenance and adaptation of form in trabecular bone. Nature 405:704-706.

Hutchinson JR. 2002. The evolution of hindlimb tendons and muscles on the line to crown-group birds. Comparative Biochemistry and Physiology, Part A 133:1051-1086.

Hutchinson JR. 2004. Biomechanical Modeling and Sensitivity Analysis of Bipedal Running Ability. I. Extant Taxa. Journal of Morphology 262:421-440.

Hutchinson JR, and Allen V. 2009. The evolutionary continuum of limb function from early theropods to birds. Naturwissenschaften 96:423-448. 
1394

1395

1396

1397

1398

1399

1400

1401

1402

1403

1404

1405

1406

1407

1408

1409

1410

1411

1412

1413

1414

1415

1416

1417

1418

1419

1420

1421

1422

1423

1424

1425

1426

1427

1428

1429

1430

1431

1432

1433

1434

1435

1436

1437

1438

1439
Hutchinson JR, Anderson FC, Blemker SS, and Delp SL. 2005. Analysis of hindlimb muscle moment arms in Tyrannosaurus rex using a three-dimensional musculoskeletal computer model: implications for stance, gait, and speed. Paleobiology 31:676-701.

Hutchinson JR, and Gatesy SM. 2000. Adductors, abductors, and the evolution of archosaur locomotion. Paleobiology 26:734-751.

Hutchinson JR, Miller CE, Fritsch G, and Hildebrandt T. 2008. The Anatomical Foundation for Multidisciplinary Studies of Animal Limb Function: Examples from Dinosaur and Elephant Limb Imaging Studies. In: Endo H, and Frey R, eds. Anatomical Imaging: Towards a New Morphology. Tokyo: Springer, 23-38.

Hutchinson JR, Rankin JW, Rubenson J, Rosenbluth KH, Siston RA, and Delp SL. 2015. Musculoskeletal Modelling of an ostrich (Stuthio camelus) pelvic limb: influence of limb orientation of muscular capacity during locomotion. PeerJ 3:e1001.

Jacobs CR. 2000. The mechanobioogy of cancellous bone structural adaptation. Journal of Rehabilitation Research and Development 37:209-216.

Jacobs CR, Simo JC, Beaupré GS, and Carter DR. 1997. Adaptive bone remodeling incorporating simultaneous density and anisotropy considerations. Journal of Biomechanics 30:603-613.

Jacobson RD, and Hollyday M. 1982. A Behavioural and Electromyographic Study of Walking in the Chick. Journal of Neurophysiology 48:238-256.

Jang IG, and Kim IY. 2008. Computational study of Wolff's law with trabecular architecture in the human proximal femur using topology optimization. Journal of Biomechanics 41:2353-2361.

Jang IG, and Kim IY. 2010a. Application of design space optimization to bone remodeling simulation of trabecular architecture in human proximal femur for higher computational efficiency. Finite Elements in Analysis and Design 46:311-319.

Jang IG, and Kim IY. 2010b. Computational simulation of cortical and trabecular bone change in human proximal femur during bone remodelling. Journal of Biomechanics 43:294-301.

Kabel J, van Rietbergen B, Odgaard A, and Huiskes R. 1999. Constitutive Relationships of Fabric, Density, and Elastic Properties in Cancellous Bone Architecture. Bone 25:481486.

Kambic RE, Roberts TJ, and Gatesy SM. 2014. Long-axis rotation: a missing degree of freedom in avian bipedal locomotion. Journal of Experimental Biology 217:2770-2782.

Kazemi M, Dabiri Y, and Li LP. 2013. Recent Advances in Computational Mechanics of the Human Knee Joint. Computational and Mathematical Methods in Medicine 2013:718423.

Kivell TL. 2016. A review of trabecular bone functional adaptation: what have we learned from trabecular analyses in extant hominoids and what can we apply to fossils? Journal of Anatomy 228:569-594.

Koch JC. 1917. The laws of bone architecture. American Journal of Anatomy 21:177-298.

Lamas LP, Main RP, and Hutchinson JR. 2014. Ontogenetic scaling patterns and functional anatomy of the pelvic limb musculature in emus (Dromaius novaehollandiae). PeerJ 2:e716.

Lanyon LE. 1974. Experimental support for the trajectorial theory of bone structure. Journal of Bone and Joint Surgery 56B:160-166.

Lanyon LE. 1996. Locomotor Loading and Functional Adaptation in Limb Bones. Symposium of the Zoological Society of London 48:305-329. 
1440

1441

1442

1443

1444

1445

1446

1447

1448

1449

1450

1451

1452

1453

1454

1455

1456

1457

1458

1459

1460

1461

1462

1463

1464

1465

1466

1467

1468

1469

1470

1471

1472

1473

1474

1475

1476

1477

1478

1479

1480

1481

1482

1483

1484

1485

Lerner ZF, Gadomski BC, Ipson AK, Haussler KK, Puttlitz CM, and Browning RC. 2015. Modulating Tibiofemoral Contact Force in the Sheep Hind Limb via Treadmill Walking: Predictions From an Opensim Musculoskeletal Model. Journal of Orthopaedic Research 33:1128-1133.

Liao L. 2011. A Study of Inertia Relief Analysis. 52nd AIAA/ASME/ASCE/AHS/ASC Structures, Structural Dynamics and Materials Conference. Denver. p AIAA 2011-2002.

Lovejoy CO. 2004. The natural history of human gait and posture. Part 2. Hip and thigh. Gait \& Posture 21:113-124.

Lovejoy CO, Meindl RS, Ohman JC, Heiple KG, and White TD. 2002. The Maka Femur and Its Bearing on the Antiquity of Human Walking: Applying Contemporary Concepts of Morphogenesis to the Human Fossil Record. American Journal of Physical Anthropology 119:97-133.

MacLatchy L, and Müller R. 2002. A comparison of the femoral head and neck trabecular architecture of Galago and Perodicticus using micro-computed tomography $(\mu \mathrm{CT})$. Journal of Human Evolution 43:89-105.

Maga M, Kappelman J, Ryan TM, and Ketcham RA. 2006. Preliminary Observations on the Calcaneal Trabecular Microarchitecture of Extant Large-Bodied Hominoids. American Journal of Physical Anthropology 129:410-417.

Main RP, and Biewener AA. 2007. Skeletal strain patterns and growth in the emu hindlimb during ontogeny. Journal of Experimental Biology 210:2676-2690.

Marsh RL, Ellerby DJ, Carr JA, Henry HT, and Buchanan CI. 2004. Partitioning the Energetics of Walking and Running: Swinging the Limbs is Expensive. Science 303:80-83.

Martelli S, Taddei F, Testi D, Delp SL, and Viceconti M. 2011. NMSBuilder: an application to personalize NMS models. Proceedings of the 23rd Congress of the International Society of Biomechanics. Brussels.

Matarazzo SA. 2015. Trabecular Architecture of the Manual Elements Reflects Locomotor Patterns in Primates. PLoS ONE 10:e0120436.

McGowan C. 1979. The Hind Limb Musculature of the brown Kiwi, Apteryx australis mantelli. Journal of Morphology 160:33-74.

McHenry CR, Wroe S, Clausen PD, Moreno K, and Cunningham E. 2007. Supermodeled sabercat, predatory behaviour in Smilodon fatalis revealed by high-resolution 3D computer simulation. Proceedings of the National Academy of Sciences 104:1601016015.

Medler S. 2002. Comparative trends in shortening velocity and force production in skeletal muscle. American Journal of Physiology 283:R368-R378.

Miller Z, Fuchs MB, and Arcan M. 2002. Trabecular bone adaptation with an orthotropic material model. Journal of Biomechanics 35:247-256.

Modenese L, and Phillips ATM. 2012. Prediction of hip contact forces and muscle activations during walking at different speeds. Multibody System Dynamics 28:157-168.

Muir GD, Gosline JM, and Steeves JD. 1996. Ontogeny of bipedal locomotion: walking and running in the chick. Journal of Physiology 493:589-601.

Mullender MG, and Huiskes R. 1995. Proposal for the Regulatory Mechanism of Wolff's Law. Journal of Orthopaedic Research 13:503-512.

O'Neill MC, Lee L-F, Larson SG, Demes B, Stern JT, Jr, and Umberger BR. 2013. A threedimensional musculoskeletal model of the chimpanzee (Pan troglodytes) pelvis and hindlimb. Journal of Experimental Biology 216:3709-3723. 
1486

1487

1488

1489

1490

1491

1492

1493

1494

1495

1496

1497

1498

1499

1500

1501

1502

1503

1504

1505

1506

1507

1508

1509

1510

1511

1512

1513

1514

1515

1516

1517

1518

1519

1520

1521

1522

1523

1524

1525

1526

1527

1528

1529
Odgaard A, Kabel J, van Rietbergen B, Dalstra M, and Huiskes R. 1997. Fabric and elastic principal directions of cancellous bone are closely related. Journal of Biomechanics 30:487-495.

Page AE, Allan C, Jasty M, Harrigan TP, Bragdon CR, and Harris WH. 1993. Determination of loading parameters in the canine hip in vivo. Journal of Biomechanics 26.

Patak AE, and Baldwin J. 1998. Pelvis Limb Musculature in the Emu Dromaius novaehollandiae (Aves: Struthioniformes: Dromaiidae): Adaptations to High-Speed Running. Journal of Morphology 238:23-37.

Pauwels F. 1980. Biomechanics of the Locomotor Apparatus. Berlin: Springer-Verlag.

Paxton H, Anthony NB, Corr SA, and Hutchinson JR. 2010. The effects of selective breeding on the architectural properties of the pelvic limb in broiler chickens: a comparative study across modern and ancestral populations. Journal of Anatomy 217:153-166.

Pedotti A, Krishnan VV, and Stark L. 1978. Optimization of Muscle-Force Sequencing in Human Locomotion. Mathematical Biosciences 38:57-76.

Phillips ATM, Villette CC, and Modenese L. 2015. Femoral bone mesoscale structural architecture prediction using musculoskeletal and finite element modelling. International Biomechanics 2:43-61.

Polk JD, Blumenfeld J, and Ahluwalia D. 2008. Knee Posture Predicted From Subchondral Apparent Density in the Distal Femur: An Experimental Validation. The Anatomical Record 16:323-329.

Pontzer H, Lieberman DE, Momin E, Devlin MJ, Polk JD, Hallgrímsson B, and Cooper DML. 2006. Trabecular bone in the bird knee responds with high sensitivity to changes in load orientation. Journal of Experimental Biology 209:57-65.

Radin EL, Orr RB, Kelman JL, Paul IL, and Rose RM. 1982. Effect of prolonged walking on concrete on the knees of sheep. Journal of Biomechanics 15:487-492.

Rankin JW, Rubenson J, and Hutchinson JR. 2016. Inferring muscle functional roles of the ostrich pelvic limb during walking and running using computer optimizaion. Journal of the Royal Society Interface 13:20160035.

Reed KL, and Brown TD. 2001. Elastic modulus and strngth of emu cortical bone. The Iowa Orthopaedic Journal 21:53-57.

Regnault S, Allen VR, Chadwick KP, and Hutchinson JR. 2017. Analysis of the moment arks and kinematics of ostrich (Struthio camelus) double patellar sesamoids. Journal of Experimental Zoology 327:163-171.

Reilly SM. 2000. Locomotion in the Quail (Coturnix japonica): The Kinematics of Walking and Increasing Speed. Journal of Morphology 243:173-185.

Ren L, Miller CE, Lair R, and Hutchinson JR. 2010. Integration of biomechanical compliance, leverage, and power in elephant limbs. Proceedings of the National Academy of Sciences 107:7078-7082.

Richmond BG, Nakatsukasa M, Griffin NL, Ogihara N, and Ketcham RA. 2005. Trabecular bone structure in a bipedally trained macaque. Seventy-Fourth Annual Meeting of the American Association of Physical Anthropologists, April 6-9, 2005. Milwaukee, United States of America. p 175-176.

Roberts TJ, Chen MS, and Taylor CR. 1998. Energetics of bipedal running. II. Limb design and running mechanics. Journal of Experimental Biology 205:2753-2762. 
1530

1531

1532

1533

1534

1535

1536

1537

1538

1539

1540

1541

1542

1543

1544

1545

1546

1547

1548

1549

1550

1551

1552

1553

1554

1555

1556

1557

1558

1559

1560

1561

1562

1563

1564

1565

1566

1567

1568

1569

1570

1571

1572

1573

1574

1575
Rose KA, Bates KT, Nudds RL, and Codd JR. 2016. Ontogeny of sex differences in the energetics and kinematics of terrestrial locomotion in leghorn chickens (Gallus gallus domesticus). Scientific Reports 6:24292.

Rubenson J, Lloyd DG, Besier TF, Heliams DB, and Fournier PA. 2007. Running in ostriches (Stuthio camelus): three-dimensional joint axes alignment and joint kinematics. Journal of Experimental Biology 210:2548-2562.

Rubenson J, Lloyd DG, Heliams DB, Besier TF, and Fournier PA. 2011. Adaptations for economical bipedal running: the effect of limb structure on three-dimensional joint mechanics. Journal of the Royal Society Interface 8:740-755.

Rudman KE, Aspden RM, and Meakin JR. 2006. Compression or tension? The stress distribution in the proximal femur. BioMedical Engineering OnLine 5:12.

Ruimerman R, Hilbers P, van Rietbergen B, and Huiskes R. 2005. A theoretical framework for strain-related trabecular bone maintenance and adaptation. Journal of Biomechanics 38:931-941.

Ryan TM, and Ketcham RA. 2002. The three-dimensional structure of trabecular bone in the femoral head of strepsirrhine primates. Journal of Human Evolution 43:1-26.

Ryan TM, and Ketcham RA. 2005. Angular Orientation of Trabecular Bone in the Femoral Head and Its Relationship to Hip Joint Loads in Leaping Primates. Journal of Morphology 265:249-263.

Ryan TM, and Shaw CN. 2012. Unique Suites of Trabecular Bone Features Characterize Locomotor Behavior in Human and Non-Human Anthropoid Primates. PLoS ONE 7:e41037.

Schaller NU, D'Août K, Villa R, Herkner B, and Aerts P. 2011. Toe function and dynamic pressure distribution in ostrich locomotion. Journal of Experimental Biology 214:11231130.

Sheffield KM, and Blob RW. 2011. Loading mechanics of the femur in tiger salamanders (Ambystoma tigrinum) during terrestrial locomotion. Journal of Experimental Biology 214:2603-2615.

Smith NC, Wilson AM, Jespers KJ, and Payne RC. 2006. Muscle architecture and functional anatomy of the pelvic limb of the ostrich (Struthio camelus). Journal of Anatomy 209:765-779.

Steele KM, DeMers MS, Schwartz MH, and Delp SL. 2012. Compressive tibiofemoral force during crouch gait. Gait \& Posture 35:556-560.

Stoessel A, and Fischer MS. 2012. Comparative intralimb coordination in avian bipedal locomotion. Journal of Experimental Biology 215:4055-4069.

Stops A, Wilcox R, and Jin Z. 2012. Computational modelling of the natural hip: a review of finite element and multibody simulations. Computer Methods in Biomechanics and Biomedical Engineering 15:963-979.

Su A, Wallace IJ, and Nakatsukasa M. 2013. Trabecular bone anisotropy and orientation in an Early Pleistocene hominin talus from East Turkana, Kenya. Journal of Human Evolution 64:667-677.

Su SC, Skedros JG, Bachus KN, and Bloebaum RD. 1999. Loading conditions and cortical bone construction of an artiodactyl calcaneus. Journal of Experimental Biology 202:32393254.

Sverdlova N. 2011. Tensile trabeculae - Myth or Reality? Journal of Musculoskeletal and Neuronal Interactions 11:1-7. 
1576

1577

1578

1579

1580

1581

1582

1583

1584

1585

1586

1587

1588

1589

1590

1591

1592

1593

1594

1595

1596

1597

1598

1599

1600

1601

1602

1603

1604

1605

1606

1607

1608

1609

1610

1611

1612

1613

1614

1615

1616

1617

1618

1619

1620

1621
Taylor SJG, and Walker PS. 2001. Forces and moments telemetered from two distal femoral replacements during various activities. Journal of Biomechanics 34:839-848.

Tsegai ZJ, Kivell TL, Gross T, Nguyen NH, Pahr DH, Smaers JB, and Skinner MM. 2013. Trabecular Bone Structure Correlates with Hand Posture and Use in Hominoids. PLoS ONE 8:e78781.

Tsubota K, Adachi T, and Tomita Y. 2002. Functional adaptation of cancellous bone in human proximal femur predicted by trabecular surface remodeling simulation toward uniform stress state. Journal of Biomechanics 35:1541-1551.

Tsubota K, Suzuki Y, Yamada T, Hojo M, Makinouchi A, and Adachi T. 2009. Computer simulation of trabecular remodelling in human proximal femur using large-scale voxel FE models: Approach to understanding Wolff's law. Journal of Biomechanics 42:10881094.

Turner CH. 1998. Three Rules for Bone Adaptation to Mechanical Stimuli. Bone 23:399-407.

Turner CH, Anne V, and Pidaparti RMV. 1997. A uniform strain criterion for trabecular bone adaptation: do continuum-level strain gradients drive adaptation? Journal of Biomechanics 30:555-563.

Turner CH, Cowin SC, Rho JY, Ashman RB, and Rice JC. 1990. The fabric dependence of the orthotropic elastic constants of cancellous bone. Journal of Biomechanics 23:549-561.

Ulrich D, van Rietbergen B, Laib A, and Rüegsegger P. 1999. The Ability of Three-Dimensional Structural Indices to Reflect Mechanical Aspects of Trabecular Bone. Bone 25:55-60.

Valente G, Pitto L, Testi D, Seth A, Delp SL, Stagni R, Viceconti M, and Taddei F. 2014. Are Subject-Specific Musculoskeletal Models Robust to the Uncertainties in Parameter Identification? PLoS ONE 9:e112625.

van Arkel RJ, Modenese L, Phillips ATM, and Jeffers JRT. 2013. Hip Abduction Can Prevent Posterior Edge Loading of Hip Replacements. Journal of Orthopaedic Research 31:11721179.

van der Meulen MCH, Morgan TG, Yang X, Baldini TH, Myers ER, Wright TM, and Bostrom MPG. 2006. Cancellous bone adaptation to in vivo loading in a rabbit model. Bone 38:871-877.

van der Meulen MCH, Yang X, Morgan TG, and Bostrom MPG. 2009. The Effects of Loading on Cancellous Bone in the Rabbit. Clinical Orthopaedics and Related Research 467:2000-2006.

Vander Sloten J, and Van der Perre G. 1989. Trabecular structure compared to stress trajectories in the proximal femur and calcaneus. Journal of Biomedical Engineering 11:203-208.

Verner KA, Lehner M, Lamas LP, and Main RP. 2016. Experimental tests of planar strain theory for predicting cross-sectional longitudinal and shear strains. Journal of Experimental Biology 219:3082-3090.

Volpato V, Viola TB, Nakatsusaka M, Bondioli L, and Macchiarelli R. 2008. Textural characteristics of the iliac-femoral trabecular pattern in a bipedally trained Japanese macaque. Primates 49:16-25.

Wang H, Ji B, Liu XS, Guo XE, Huang Y, and Hwang K-C. 2012. Analysis of microstructural and mechanical alterations of trabecular bone in a simulated three-dimensional remodelling process. Journal of Biomechanics 45:2417-2425.

Wink CS, Elsey RM, St Onge M, and Zimny MI. 1989. Neural Element in the Cruciate Ligaments and Menisci of the Knee Joint of the American Alligator, Alligator mississippiensis. Journal of Morphology 202:165-172. 
1622

1623

1624

1625

1626

1627

1628

1629

1630

1631

1632

1633

1634

1635

1636

1637

1638

1639

1640

1641

1642

1643

1644

1645

1646

1647

1648

1649

1650

1651

1652

1653

1654

1655

1656

Winter DA. 2009. Biomechanics and Motor Control of Human Movement. Hoboken: John Wiley \& Sons, Inc.

Witte TH, Knill K, and Wilson AM. 2004. Determination of peak vertical ground reaction force from duty factor in the horse (Equus caballus). Journal of Experimental Biology 207:3639-3648.

Wolff J. 1892. Das Gesetz der Transformation der Knochen. Berlin: August Hirschwald. Wroe S, Moreno K, Clausen PD, McHenry CR, and Curnoe D. 2007. High-Resolution ThreeDimensional Computer Simulation of Hominid Cranial Mechanics. The Anatomical Record 290:1248-1255.

Zadpoor AA, Campoli G, and Weinans H. 2013. Neural network prediction of load from the morphology of trabecular bone. Applied Mathematical Modelling 37:5260-5276.

Zinoviev AV. 2010. Comparative anatomy, structural modifications and adaptive evolution of avian apparatus of bipedal locomotion. Moscow: KMK Scientific Press Ltd.

\section{$\underline{\text { II.9 Figure captions }}$}

Figure 1. The musculoskeletal model of the chicken hindlimb developed in this study. This is shown in the 'neutral posture' for all joints, that is, when all joint angles are zero. (A-C) Geometries of the musculotendon actuators in relation to the bones, in lateral (A), anterior (B) and oblique anterolateral (C) views. (D-E) Location and orientation of joint coordinate systems (red, green and blue axes), the centres of mass for each segment (grey and white balls) and the soft tissue volumes, derived from $\mathrm{CT}$ scans and used to calculate mass properties; these are shown in the same views as A-C. Also reported in F are the masses for each segment; the pelvis segment represents the body as well as the contralateral limb. In D-F, the flexion-extension axis of each joint is the blue axis. For scale, the length of each arrow in the triad of the global coordinate system is $40 \mathrm{~mm}$.

Figure 2. Musculoskeletal simulation of a given test posture. Muscles that are active are red, whilst those set to be inactive during simulation are blue. External loads applied to the pes segment are the vertical ground reaction force (GRF) and moments about the $x$ and $y$ axes ( $M_{x}$ and $M_{y}$, respectively). A reserve actuator is also applied to the metatarsophalangeal joint (purple). Loads are not shown to scale. 
Figure 3. Geometry, forces and constraints involved in the finite element analysis of a given test posture. (A, B) For each posture, two simulations were performed, one for the femur (A) and one

1661

1662

1663

1664

1665

1666

1667

1668

1669

1670

1671

1672

1673

1674

1675

1676

1677

1678

1679

1680

1681

1682

1683

1684

1685

1686 for the tibiotarsus + fibula (B). Muscle and ligament forces are red, segment weights are blue, joint forces are green and joint moments are orange. The focal bones in each simulation were 'bookended' between their adjacent articulating bones, to which restraints or joint forces were applied. (C) The intervening soft tissues between focal bones and their neighbouring bones were modelled as a single homogenous volume (turquoise). (D) Knee joint forces were applied as a remote force: the force was applied to a remote point (knee joint centre, red dot), which was topologically attached to a neighbouring bone via constraint equations (red lines, schematic illustration only). Loads are not shown to scale.

Figure 4. The postures tested for in the chicken. Around the periphery are the different postures tested, shown in lateral view, with the final solution posture in the centre box, shown in lateral, dorsal and anterior views; the whole-body COM location is also shown for the solution posture in lateral view. Joint angles for each posture are given in blue font; hip joint angles are given in the order of flexion-extension, abduction-adduction and long-axis rotation. Hip extension angle is expressed relative to the horizontal, whereas knee and ankle angles are expressed relative to the femur and tibiotarsus (respectively). For the other hip angles, positive values indicate abduction and external rotation, whereas negative values indicate adduction and internal rotation. The metatarsophalangeal joint angle is expressed relative to the neutral posture. The angular deviation between $\boldsymbol{\sigma}_{3}$ and $\mathbf{u}_{1}$ for each posture is also given in red font (reported as femoral head, then medial femoral condyle). The solution posture resulted in the greatest degree of overall correspondence between principal stress trajectories and observed cancellous bone architectural patterns in birds, as assessed by qualitative comparisons across the femur, tibiotarsus and fibula, as well as quantitative results for the femoral head and medial femoral condyle. 
1687 Figure 5. Principal stress trajectories for the femur in the solution posture compared to

1688

1689

1690

1691

1692

1693

1694

1695

1696

1697

1698

1699

1700

1701

1702

1703

1704

1705

1706

1707

1708

1709

1710

1711

1712

1713

1714

1715

1716

1717

cancellous bone fabric. (A, C, E, G, I, K, M, O) Stress vector fields ( $\sigma_{3}$ in all cases) compared to exemplar fabric vector fields for birds (B, D, F, H, J, L, N, P, $\mathbf{u}_{1}$ in all cases; cf. Figs 16 and 24 of Part I), plotted on translucent renderings of the bone; not to scale. For easier visual comparison, the stress trajectories were 'downsampled' in a custom MATLAB script, by interpolating the raw stress results at each finite element node to a regular grid. (A - D) In the femoral head, in anterior $(\mathrm{A}, \mathrm{B})$ and medial $(\mathrm{C}, \mathrm{D})$ views. $(\mathrm{E}-\mathrm{H})$ Under the facies antitrochanterica, in anterior $(\mathrm{E}, \mathrm{F})$ and lateral $(\mathrm{G}, \mathrm{H})$ views. (I - L) In the trochanteric crest, in anterior $(\mathrm{I}, \mathrm{J})$ and lateral $(\mathrm{K}, \mathrm{L})$ views. $(\mathrm{M}, \mathrm{N})$ Medial femoral condyle, parallel to the sagittal plane and in medial view. $(\mathrm{O}, \mathrm{P})$ Lateral femoral condyle, parallel to the sagittal plane and in lateral view. (Q) Comparison of the mean direction of $\boldsymbol{\sigma}_{3}$ (blue) in the femoral head and the mean direction of $\mathbf{u}_{1}$ (red) for birds, plotted on an equal-angle stereoplot, with northern hemisphere projection (using StereoNet 9.5;

Allmendinger et al. 2013; Cardozo \& Allmendinger 2013). (R) Comparison of the mean direction of $\boldsymbol{\sigma}_{3}$ in the medial femoral condyle and the mean direction of $\mathbf{u}_{1}$ for birds, plotted on an equalangle stereoplot, with southern hemisphere projection. Insets in Q and R show locations of regions for which the mean direction of $\boldsymbol{\sigma}_{3}$ was calculated. The orange dots in $\mathrm{Q}$ and $\mathrm{R}$ indicate the mean direction of $\boldsymbol{\sigma}_{3}$ for the muscle force sensitivity test; note how close these are to the original results for the solution posture.

Figure 6. Principal stress trajectories for the tibiotarsus in the solution posture compared to cancellous bone fabric. (A, C, G, I, K, M, O, Q, S) Stress vector fields ( $\boldsymbol{\sigma}_{1}$ in red, $\boldsymbol{\sigma}_{3}$ in blue) compared to exemplar fabric vector fields for birds (B, D, H, J, L, N, P, R, T, $\mathbf{u}_{1}$ in all cases; cf. Figs 31 and 36 of Part I), plotted on translucent renderings of the bone; not to scale. (A - D) Anterior cnemial crest, in anterior (A, B) and medial (C, D) views. (E, F) Vector field of $\sigma_{1}$ in the anterior cnemial crest in the muscle force sensitivity test, shown in the same views as A and C, respectively. $(\mathrm{G}-\mathrm{J})$ Lateral cnemial crest, in anterior $(\mathrm{G}, \mathrm{H})$ and lateral $(\mathrm{I}, \mathrm{J})$ views. $(\mathrm{K}-\mathrm{N})$ Under the medial articular facies, parallel to the coronal plane (K, L, posterior view) and sagittal plane (M, N, medial view). $(\mathrm{O}-\mathrm{R})$ Under the lateral articular facies, parallel to the coronal plane (O, P, posterior view) and sagittal plane (Q, R, lateral view). (S, T) A 3-D slice through the middle of the proximal metaphysis, parallel to the sagittal plane; schematic insets show the 
1718 double-arcuate pattern present in both the stress trajectories and fabric vectors. (U, V) Vector

1719 field of $\boldsymbol{\sigma}_{3}$ in the articular condyles (purple = lateral condyle, pink = medial condyle) of the distal

1720 tibiotarsus, shown for 3-D slices through the middle of the condyles, in oblique anterolateral (U)

1721 and anteromedial (V) views.

1722

1723

1724 Figure 7. Principal stress trajectories for the fibula in the solution posture compared to cancellous

1725 bone fabric. (A) Vector field of $\sigma_{1}$ in the medial side of the fibular head plotted on a translucent

1726 rendering of the bone, in medial view (reversed). (B) Vector field of $\sigma_{3}$ in lateral side of the

1727 fibular head, in lateral view. (C, D) Exemplar fabric vector fields $\left(\mathbf{u}_{1}\right)$ for birds, in lateral view

1728 (cf. Figs 40G-K of Part I); not to scale.

1729

1730

1731 Figure 8. Additional aspects of the solution posture. (A) The trajectories of the principal stresses

$1732 \sigma_{1}$ (red) and $\sigma_{3}$ (blue) at the femoral mid-shaft, in anterior view. (B) Oblique principal stresses in

1733 the femoral mid-shaft indicate of strong torsional loading (orange arrows), with a positive sense.

1734 (C) The trajectories of $\sigma_{1}$ and $\sigma_{3}$ at the tibial mid-shaft, in anterior view; note the almost complete

1735 lack of obliquity with respect to the long-axis of the bone. (D) Activations for each muscle

1736 actuator in the musculoskeletal simulation. In the original simulation all musculotendon actuators

1737 were assigned a maximum force of 2 body weights, whilst in the sensitivity test the assigned

1738 maximum force was specific to the size and architecture of each muscle. (E) Flexion-extension

1739 muscle moment arms for the hip, knee and ankle joints; positive values indicate extension,

1740 negative values indicate flexion. For keys to abbreviations in D and E, see Table 1. 


\section{Table $\mathbf{1}$ (on next page)}

The origins and insertions of each of the muscles and ligaments represented in the chicken musculoskeletal model.

Those muscles that attach to the patella or patellar tendon were modelled as attaching in a general fashion to the apices of one of the cnemial crests (identified in brackets).

*ODF includes the flexores perforantes et perforatus digitorum II et III and flexores perforatus digitorum II, III et IV.

**ODE includes the extensores brevis digitorum III et IV and extensor proprius digiti III. 
3 Table 1. The origins and insertions of each of the muscles and ligaments represented in the chicken musculoskeletal model. Those 4 muscles that attach to the patella or patellar tendon were modelled as attaching in a general fashion to the apices of one of the cnemial crests (identified in brackets).

\begin{tabular}{|c|c|c|c|}
\hline Muscle or ligament & Abbreviation & Origin & Insertion \\
\hline Iliotibialis cranialis & IC & Anterior rim of dorsal iliac crest & Patellar tendon [medial aspect of anterior cnemial crest] \\
\hline $\begin{array}{l}\text { Iliotibialis lateralis pars } \\
\text { preacetabularis }\end{array}$ & ILPR & Dorsolateral iliac crest, anterior to acetabulum & Patellar tendon [anterior cnemial crest] \\
\hline $\begin{array}{l}\text { Iliotibialis lateralis pars } \\
\text { postacetabularis }\end{array}$ & ILPO & Dorsolateral iliac crest, posterior to acetabulum & Patellar tendon [anterior cnemial crest] \\
\hline Ambiens & AMB & Preacetabular process on proximal pubis & Lateral fibular head \\
\hline Femorotibialis externus & FMTE & Lateral femoral shaft & Patellar tendon [anterior cnemial crest] \\
\hline Femorotibialis medius & FMTM & Anterior femoral shaft & Patellar tendon [anterior cnemial crest] \\
\hline Femorotibialis internus & FMTI & Medial femoral shaft & Patellar tendon [medial aspect of anterior cnemial crest] \\
\hline Iliofibularis & ILFB & Lateral postacetabular ilium, anterior to FCLP & Fibular tubercle \\
\hline $\begin{array}{l}\text { Flexor cruris lateralis pars } \\
\text { pelvica }\end{array}$ & FCLP & $\begin{array}{l}\text { Lateral surface of posterior end of ilium and adjacent caudal } \\
\text { vertebrae }\end{array}$ & Medial proximal tibiotarsus \\
\hline $\begin{array}{l}\text { Flexor cruris lateralis pars } \\
\text { accessoria }\end{array}$ & FCLA & From FCLP & Distal posterior femur \\
\hline Flexor cruris medialis & FCM & Lateral surface of posterior end of ischium & Medial proximal tibiotarsus \\
\hline Iliofemoralis externus & IFE & Processus supratrochantericus of ilium & Trochanteric shelf of femur \\
\hline Iliofemoralis internus & IFI & Ventral preacetabular ilium, ventral to ITM & Medial surface of proximal femur (distal to femoral head) \\
\hline Iliotrochantericus cranialis & ITCR & Ventral preacetabular ilium, posterior to ITCR & Anterolateral surface of femoral trochanter \\
\hline Iliotrochantericus medius & ITM & Ventral preacetabular ilium & Anterolateral surface of femoral trochanter, distal to ITC \\
\hline Iliotrochantericus caudalis & ITC & Lateral surface of preacetabular ilium & Anterolateral surface of femoral trochanter, distal to ITC \\
\hline Ischiofemoralis & ISF & Lateral ischium & Lateral proximal femur \\
\hline $\begin{array}{l}\text { Caudofemoralis pars } \\
\text { caudalis }\end{array}$ & $\mathrm{CFC}$ & Ventrolateral surface of pygostyle & Posterior surface of proximal femoral shaft \\
\hline $\begin{array}{l}\text { Caudofemoralis pars } \\
\text { pelvica }\end{array}$ & CFP & Lateral ilium, posterior to ILFB and dorsal to ISF & Posterior surface of proximal femoral shaft (lateral to CFC) \\
\hline Obturatorius medialis & $\mathrm{OM}$ & Medial surfaces of ischium and pubis & Posterolateral surface of proximal femur \\
\hline
\end{tabular}


6 Table 1 (continued).

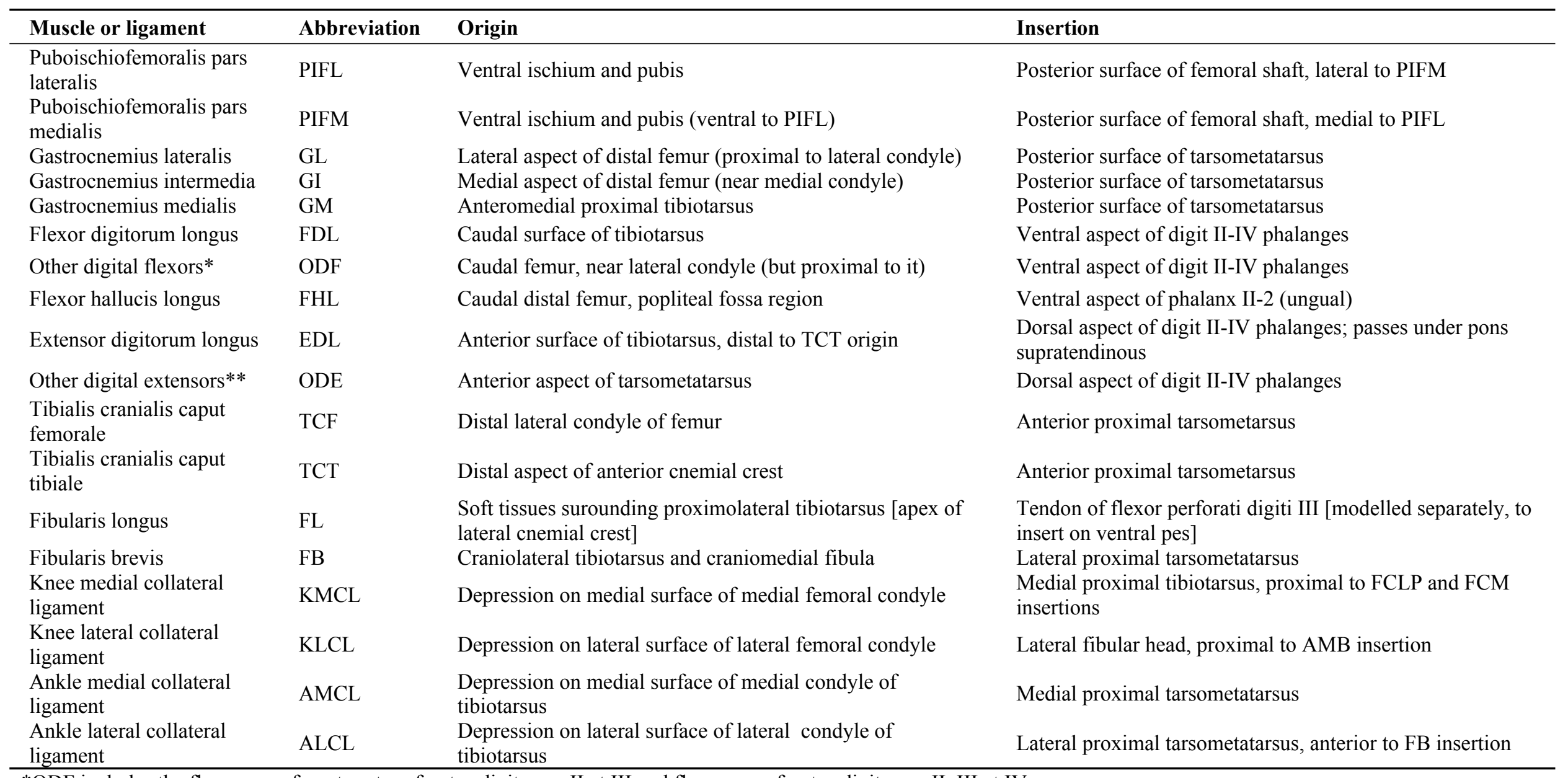

7 *ODF includes the flexores perforantes et perforatus digitorum II et III and flexores perforatus digitorum II, III et IV.

$8 * * \mathrm{ODE}$ includes the extensores brevis digitorum III et IV and extensor proprius digiti III. 


\section{Table 2 (on next page)}

Assumed activities of the muscle actuators used in the simulations.

$\mathrm{X}=$ active (capable of exerting up to $30.597 \mathrm{~N}$ of force), $\mathrm{O}=$ inactive (exerts zero force). 
1 Table 2. Assumed activities of the muscle actuators used in the simulations. $X=$ active

2 (capable of exerting up to $30.597 \mathrm{~N}$ of force), $\mathrm{O}=$ inactive (exerts zero force).

3

4

5

6

7

8

9

10

11

12

13

14

15

16

17

18

19

20

21

22

23

24

25

26

27

28

29

30

31

32

\begin{tabular}{lc}
\hline Muscle & Activity \\
\hline IC & $\mathrm{O}$ \\
ILPR & $\mathrm{O}$ \\
ILPO & $\mathrm{X}$ \\
AMB & $\mathrm{X}$ \\
FMTE & $\mathrm{X}$ \\
FMTM & $\mathrm{X}$ \\
FMTI & $\mathrm{X}$ \\
ILFB & $\mathrm{X}$ \\
FCLP & $\mathrm{X}$ \\
FCLA & $\mathrm{X}$ \\
FCM & $\mathrm{X}$ \\
IFE & $\mathrm{O}$ \\
IFI & $\mathrm{O}$ \\
ITCR & $\mathrm{O}$ \\
ITM & $\mathrm{X}$ \\
ITC & $\mathrm{X}$ \\
ISF & $\mathrm{X}$ \\
CFC & $\mathrm{X}$ \\
CFP & $\mathrm{X}$ \\
OM & $\mathrm{O}$ \\
PIFL & $\mathrm{X}$ \\
PIFM & $\mathrm{X}$ \\
GL & $\mathrm{X}$ \\
GI & $\mathrm{X}$ \\
GM & $\mathrm{X}$ \\
FDL & $\mathrm{X}$ \\
ODF & $\mathrm{X}$ \\
FHL & $\mathrm{X}$ \\
EDL & $\mathrm{O}$ \\
ODE & $\mathrm{O}$ \\
TCF & $\mathrm{O}$ \\
TCT & $\mathrm{O}$ \\
FL & $\mathrm{X}$ \\
FB & $\mathrm{X}$ \\
\hline
\end{tabular}


33

34

PeerJ reviewing PDF | (2017:12:22648:2:0:REVIEW 8 Sep 2018) 


\section{Table 3(on next page)}

Material properties used in the finite element analysis component of thesimulations.

All entities were modelled as solid, isotropic, linearly elastic materials. Values derived from Reed \& Brown (2001), Currey (2002), Erickson et al. (2002), Stops et al. (2012) and Kazemi et al. (2013), and references cited therein. *The knee soft tissues material properties reflected a composite of those of both cartilage and menisci. 
1 Table 3. Material properties used in the finite element analysis component of the 2 simulations. All entities were modelled as solid, isotropic, linearly elastic materials. Values 3 derived from Reed \& Brown (2001), Currey (2002), Erickson et al. (2002), Stops et al. 4 (2012) and Kazemi et al. (2013), and references cited therein.

\begin{tabular}{lccc}
\hline \multicolumn{1}{c}{ Material } & Density $\left(\mathbf{k g} / \mathbf{m}^{\mathbf{3}}\right)$ & Young's modulus (MPa) & Poisson's ratio \\
\hline Bone & 2,060 & 17,000 & 0.3 \\
Cartilage & 1,100 & 50 & 0.45 \\
Knee soft tissues* & 1,100 & 100 & 0.3 \\
\hline
\end{tabular}

*The knee soft tissues material properties reflected a composite of those of both cartilage and menisci. 


\section{Table 4 (on next page)}

Maximum force capability of the active muscle actuators used in the muscle force sensitivity test.

Note how the force capability can vary widely, from less than 0.1 BW (AMB) to over 6 BW (FMTI). 
1 Table 4. Maximum force capability of the active muscle actuators used in the muscle force 2 sensitivity test. Note how the force capability can vary widely, from less than $0.1 \mathrm{BW}$ 3 (AMB) to over $6 \mathrm{BW}$ (FMTI).

\begin{tabular}{lc}
\hline Muscle & $\boldsymbol{F}_{\max }(\mathbf{N})$ \\
\hline ILPO & 30.111 \\
AMB & 1.112 \\
FMTE & 19.636 \\
FMTM & 21.007 \\
FMTI & 92.110 \\
ILFB & 24.777 \\
FCLP & 20.760 \\
FCLA & 18.656 \\
FCM & 8.713 \\
ITM & 3.003 \\
ITC & 77.382 \\
ISF & 25.635 \\
CFC & 1.625 \\
CFP & 6.300 \\
PIFL & 7.981 \\
PIFM & 17.940 \\
GL & 59.539 \\
GI & 10.863 \\
GM & 71.969 \\
FDL & 31.022 \\
ODF & 58.122 \\
FHL & 22.672 \\
FL & 51.621 \\
FB & 8.737 \\
\hline &
\end{tabular}




\section{Figure 1}

The musculoskeletal model of the chicken hindlimb developed in this study.

This is shown in the 'neutral posture' for all joints, that is, when all joint angles are zero. $(A-C)$ Geometries of the musculotendon actuators in relation to the bones, in lateral $(A)$, anterior (B) and oblique anterolateral (C) views. (D-E) Location and orientation of joint coordinate systems (red, green and blue axes), the centres of mass for each segment (grey and white balls) and the soft tissue volumes, derived from CT scans and used to calculate mass properties; these are shown in the same views as A-C. Also reported in $\mathrm{F}$ are the masses for each segment; the pelvis segment represents the body as well as the contralateral limb. In D-F, the flexion-extension axis of each joint is the blue axis. For scale, the length of each arrow in the triad of the global coordinate system is $40 \mathrm{~mm}$. 
A

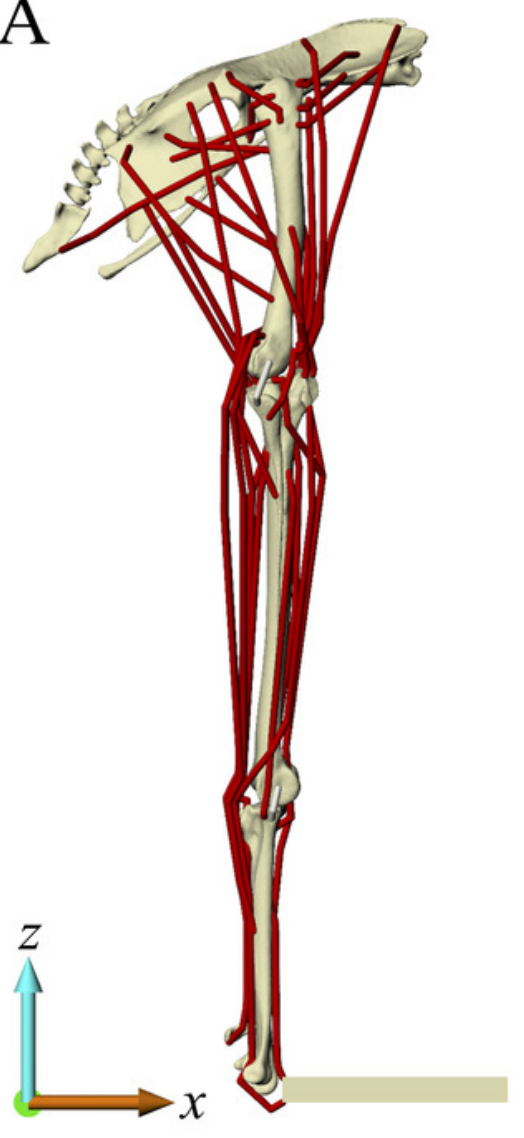

D

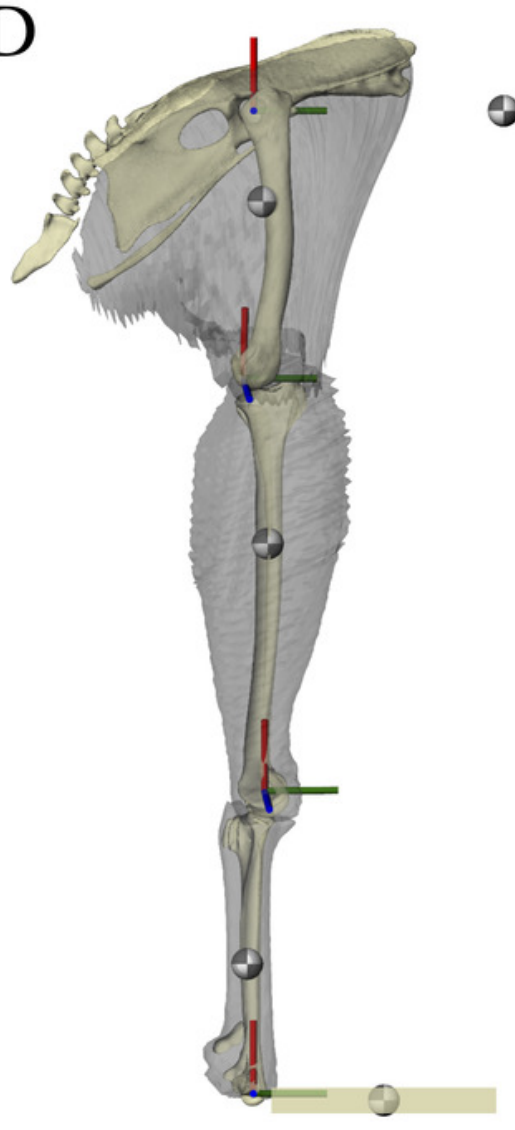

B

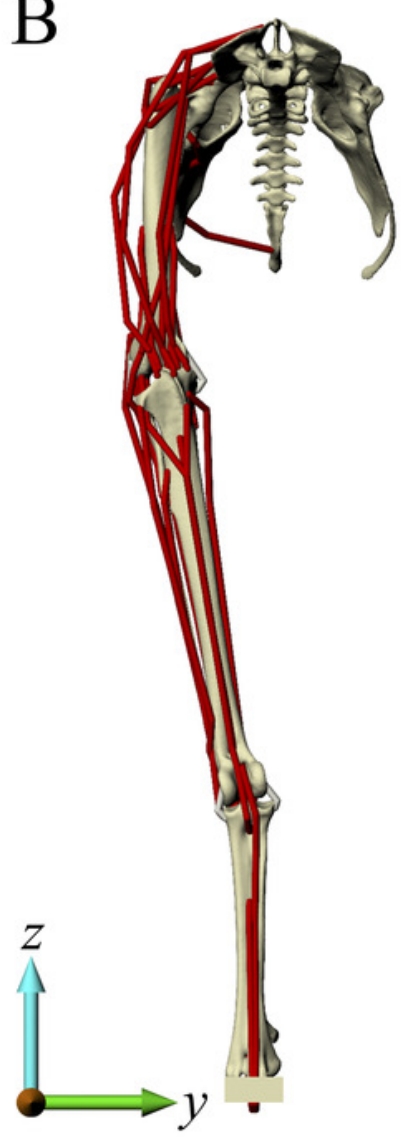

$\mathrm{E}$

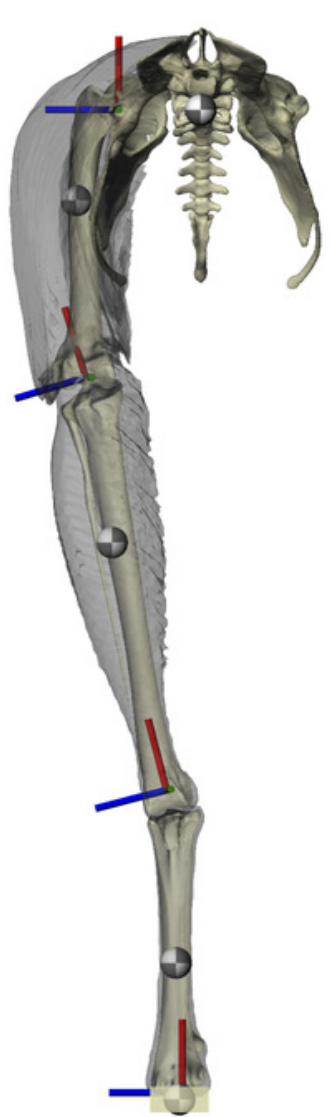

C

C
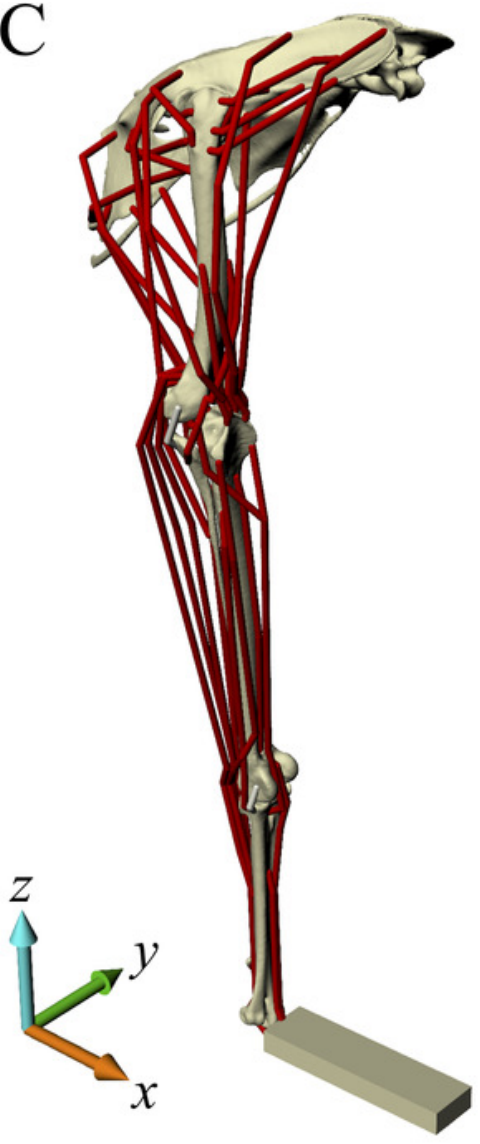

(1)

F

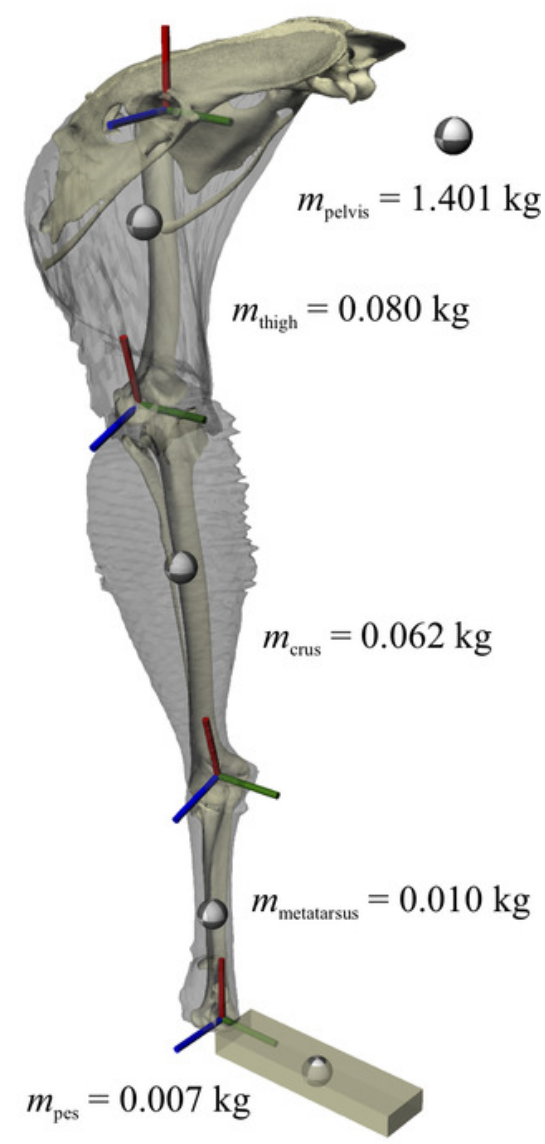


Figure 2

Musculoskeletal simulation of a given test posture.

Muscles that are active are red, whilst those set to be inactive during simulation are blue.

External loads applied to the pes segment are the vertical ground reaction force (GRF) and moments about the $x$ and $y$ axes $\left(M_{x}\right.$ and $M_{y}$, respectively). A reserve actuator is also applied to the metatarsophalangeal joint (purple). Loads are not shown to scale. 


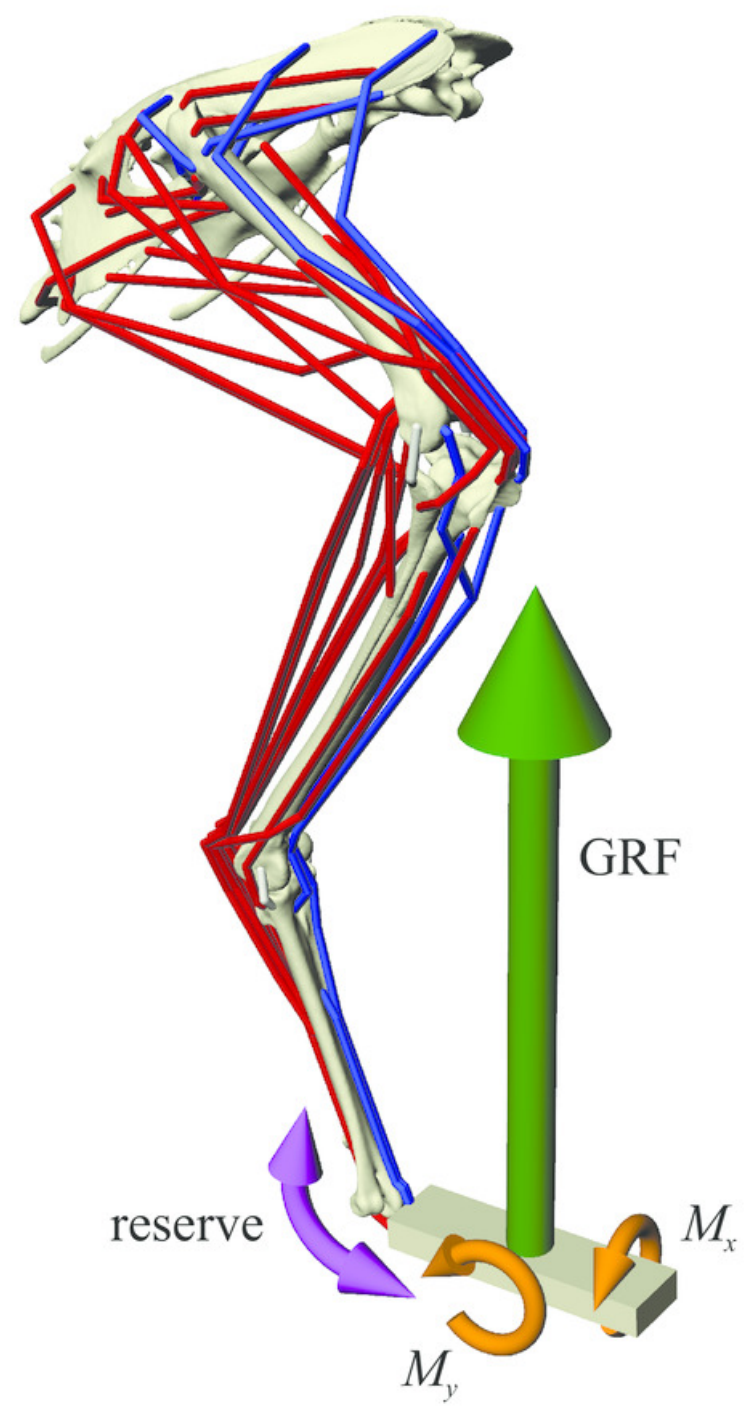




\section{Figure 3}

Geometry, forces and constraints involved in the finite element analysis of a given test posture.

(A, B) For each posture, two simulations were performed, one for the femur (A) and one for the tibiotarsus + fibula (B). Muscle and ligament forces are red, segment weights are blue, joint forces are green and joint moments are orange. The focal bones in each simulation were 'bookended' between their adjacent articulating bones, to which restraints or joint forces were applied. (C) The intervening soft tissues between focal bones and their neighbouring bones were modelled as a single homogenous volume (turquoise). (D) Knee joint forces were applied as a remote force: the force was applied to a remote point (knee joint centre, red dot), which was topologically attached to a neighbouring bone via constraint equations (red lines, schematic illustration only). Loads are not shown to scale. 

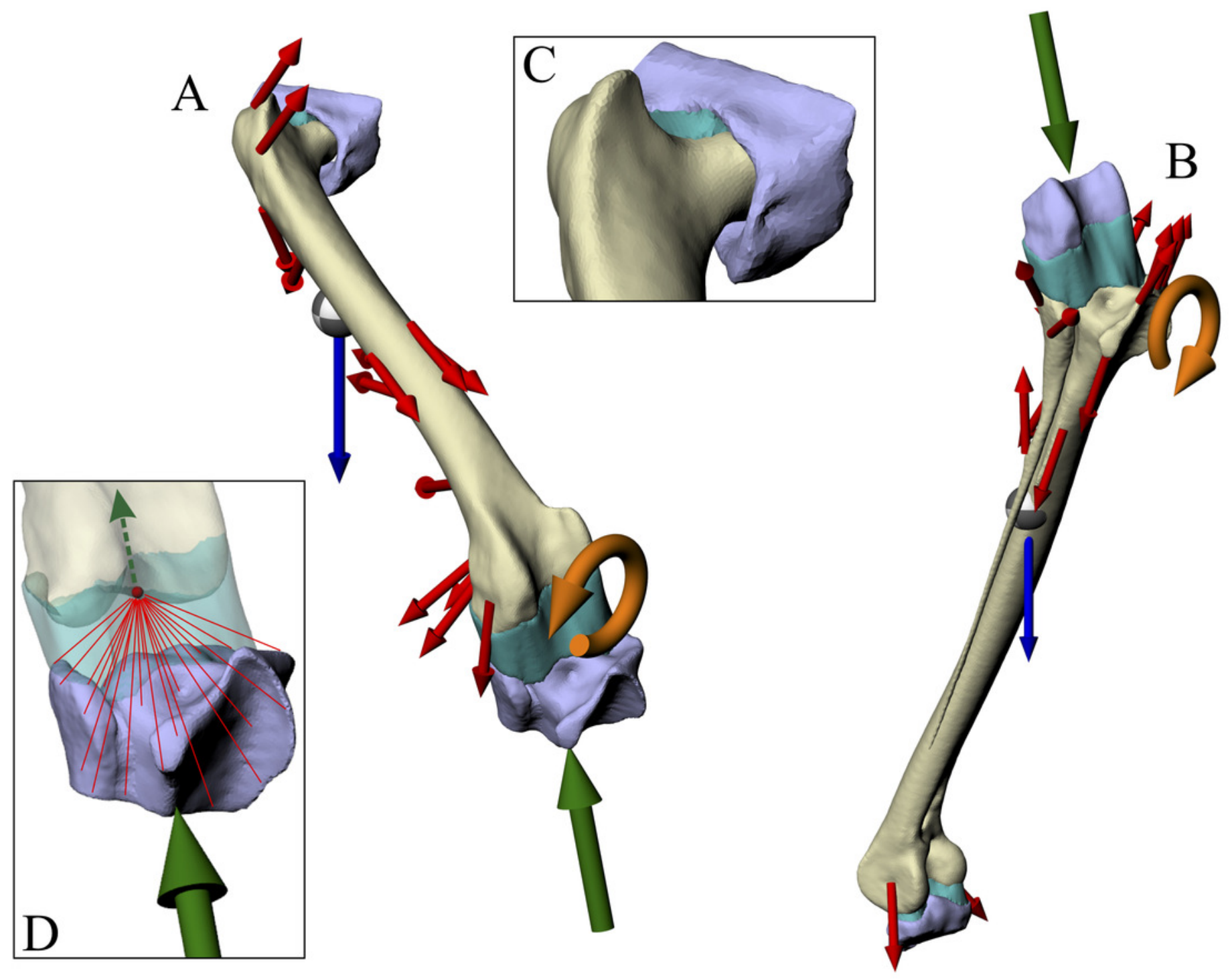


\section{Figure 4}

The postures tested for in the chicken.

Around the periphery are the different postures tested, shown in lateral view, with the final solution posture in the centre box, shown in lateral, dorsal and anterior views; the wholebody COM location is also shown for the solution posture in lateral view. Joint angles for each posture are given in blue font; hip joint angles are given in the order of flexion-extension, abduction-adduction and long-axis rotation. Hip extension angle is expressed relative to the horizontal, whereas knee and ankle angles are expressed relative to the femur and tibiotarsus (respectively). For the other hip angles, positive values indicate abduction and external rotation, whereas negative values indicate adduction and internal rotation. The metatarsophalangeal joint angle is expressed relative to the neutral posture. The angular deviation between $\boldsymbol{\sigma}_{3}$ and $\mathbf{u}_{1}$ for each posture is also given in red font (reported as femoral head, then medial femoral condyle). The solution posture resulted in the greatest degree of overall correspondence between principal stress trajectories and observed cancellous bone architectural patterns in birds, as assessed by qualitative comparisons across the femur, tibiotarsus and fibula, as well as quantitative results for the femoral head and medial femoral condyle. 


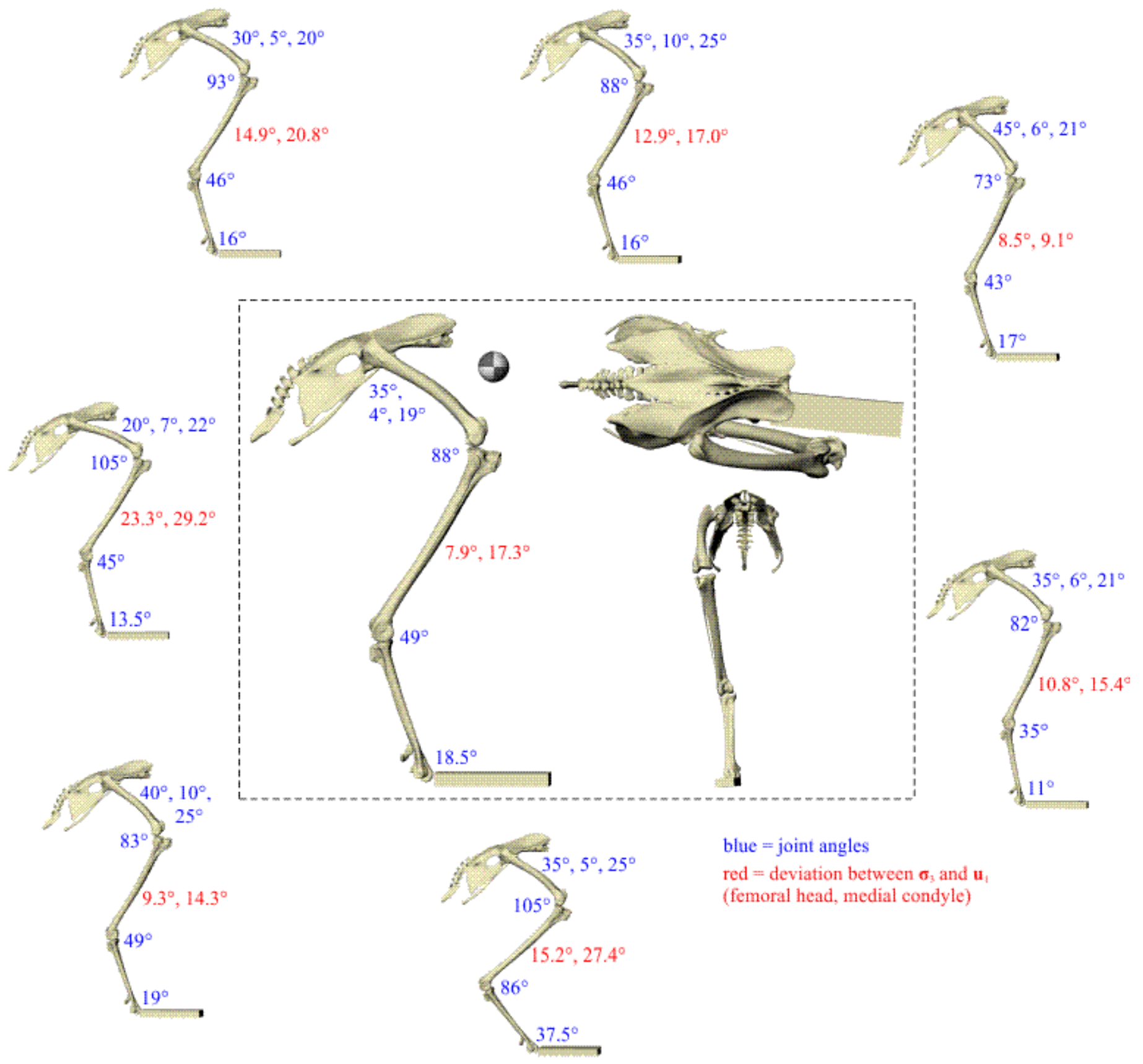




\section{Figure 5}

Principal stress trajectories for the femur in the solution posture compared to cancellous bone fabric.

(A, C, E, G, I, K, M, O) Stress vector fields ( $\boldsymbol{\sigma}_{3}$ in all cases) compared to exemplar fabric vector fields for birds ( $B, D, F, H, J, L, N, P, \mathbf{u}_{1}$ in all cases; cf. Figs 16 and 24 of Part I ), plotted on translucent renderings of the bone; not to scale. For easier visual comparison, the stress trajectories were 'downsampled' in a custom MATLAB script, by interpolating the raw stress results at each finite element node to a regular grid. (A - D) In the femoral head, in anterior (A, B) and medial (C, D) views. $(E-H)$ Under the facies antitrochanterica, in anterior $(\mathrm{E}, \mathrm{F})$ and lateral $(\mathrm{G}, \mathrm{H})$ views. $(\mathrm{I}-\mathrm{L})$ In the trochanteric crest, in anterior $(\mathrm{I}, \mathrm{J})$ and lateral $(\mathrm{K}$, L) views. (M, N) Medial femoral condyle, parallel to the sagittal plane and in medial view. ( $O$, P) Lateral femoral condyle, parallel to the sagittal plane and in lateral view. (Q) C omparison of the mean direction of $\boldsymbol{\sigma}_{3}$ (blue) in the femoral head and the mean direction of $\mathbf{u}_{1}$ (red) for birds, plotted on an equal-angle stereoplot, with northern hemisphere projection (using StereoNet 9.5; Allmendinger et al. 2013; Cardozo \& Allmendinger 2013). (R) C omparison of the mean direction of $\boldsymbol{\sigma}_{3}$ in the medial femoral condyle and the mean direction of $\mathbf{u}_{1}$ for birds, plotted on an equal-angle stereoplot, with southern hemisphere projection. Insets in Q and $\mathrm{R}$ show locations of regions for which the mean direction of $\boldsymbol{\sigma}_{3}$ was calculated. The orange dots in $\mathrm{Q}$ and $\mathrm{R}$ indicate the mean direction of $\boldsymbol{\sigma}_{3}$ for the muscle force sensitivity test; note how close these are to the original results for the solution posture. 

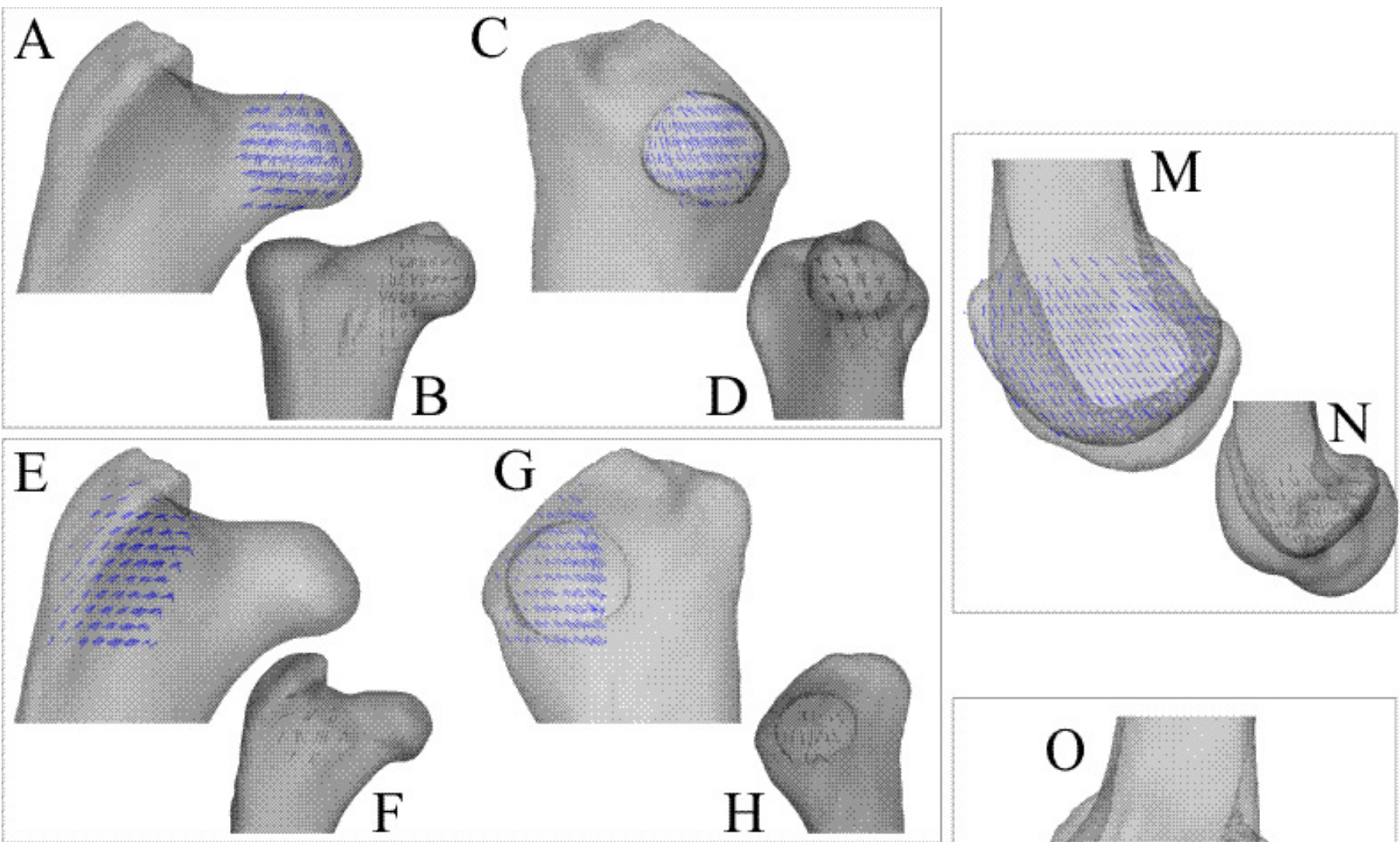

G

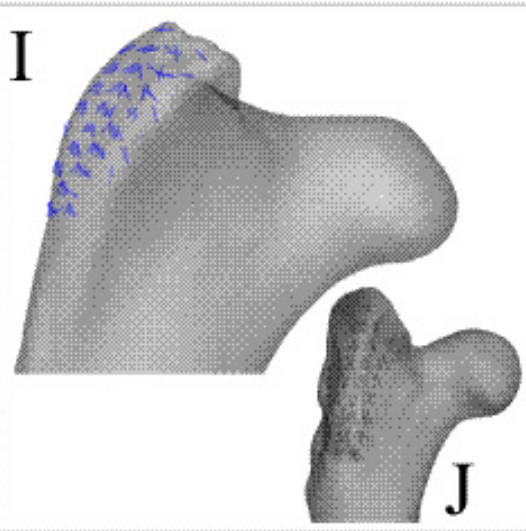

H
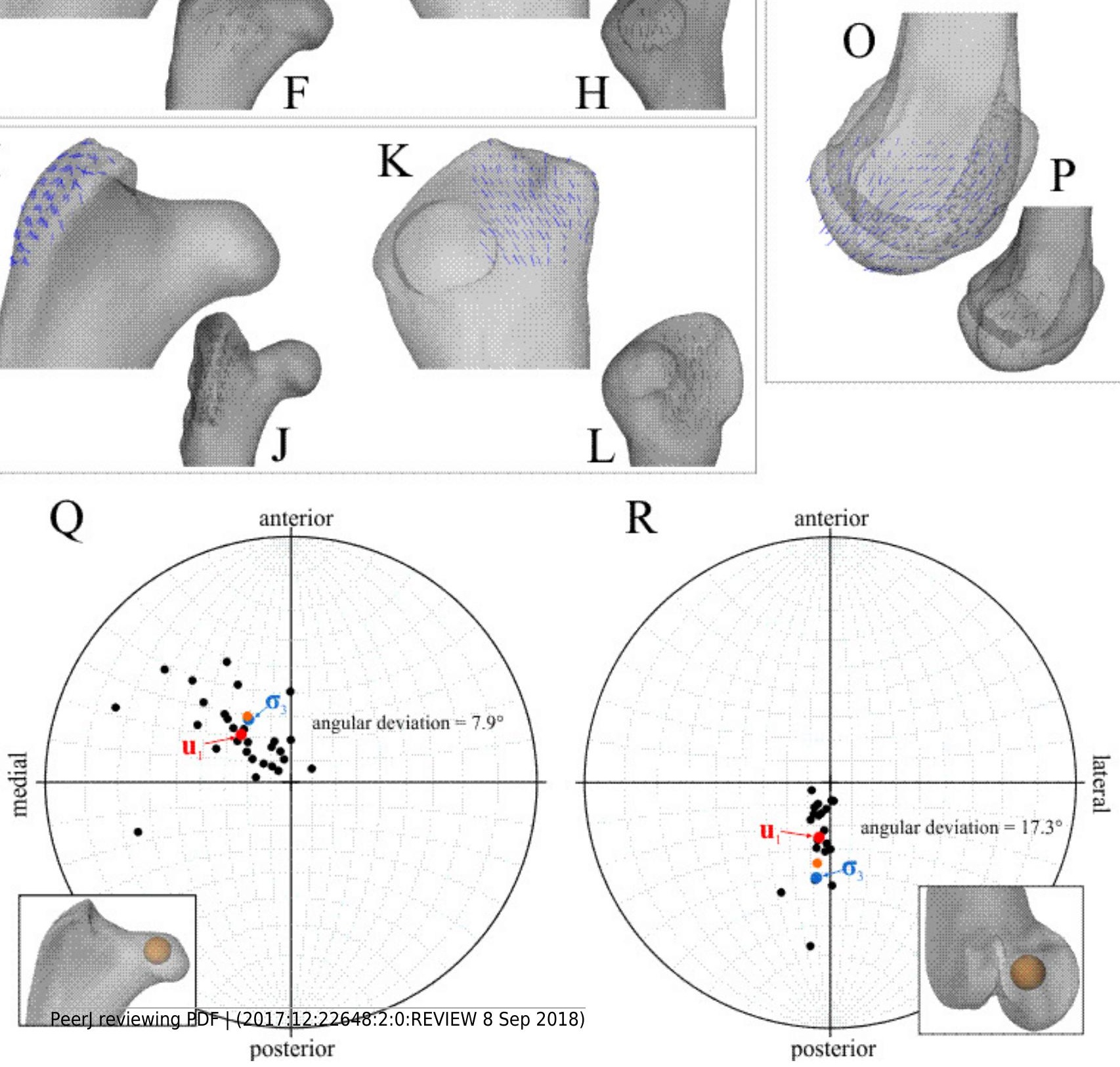


\section{Figure 6}

Principal stress trajectories for the tibiotarsus in the solution posture compared to cancellous bone fabric.

(A, C, G, I, K, M, O, Q, S) Stress vector fields ( $\boldsymbol{\sigma}_{1}$ in red, $\boldsymbol{\sigma}_{3}$ in blue) compared to exemplar fabric vector fields for birds (B, D, H, J, L, N, P, R, T, $\mathbf{u}_{1}$ in all cases; cf. Figs 31 and 36 of Part I), plotted on translucent renderings of the bone; not to scale. (A - D) Anterior cnemial crest, in anterior (A, B) and medial (C, D) views. (E, F) Vector field of $\boldsymbol{\sigma}_{1}$ in the anterior cnemial crest in the muscle force sensitivity test, shown in the same views as $A$ and $C$, respectively. ( $G$ - J) Lateral cnemial crest, in anterior $(G, H)$ and lateral $(I, J)$ views. $(K-N)$ Under the medial articular facies, parallel to the coronal plane $(\mathrm{K}, \mathrm{L}$, posterior view) and sagittal plane $(\mathrm{M}, \mathrm{N}$, medial view). (O - R) Under the lateral articular facies, parallel to the coronal plane $(\mathrm{O}, \mathrm{P}$, posterior view) and sagittal plane ( $Q, R$, lateral view). (S, T) A 3-D slice through the middle of the proximal metaphysis, parallel to the sagittal plane; schematic insets show the doublearcuate pattern present in both the stress trajectories and fabric vectors. $(U, V)$ Vector field of $\boldsymbol{\sigma}_{3}$ in the articular condyles (purple = lateral condyle, pink = medial condyle) of the distal tibiotarsus, shown for 3-D slices through the middle of the condyles, in oblique anterolateral (U) and anteromedial (V) views. 

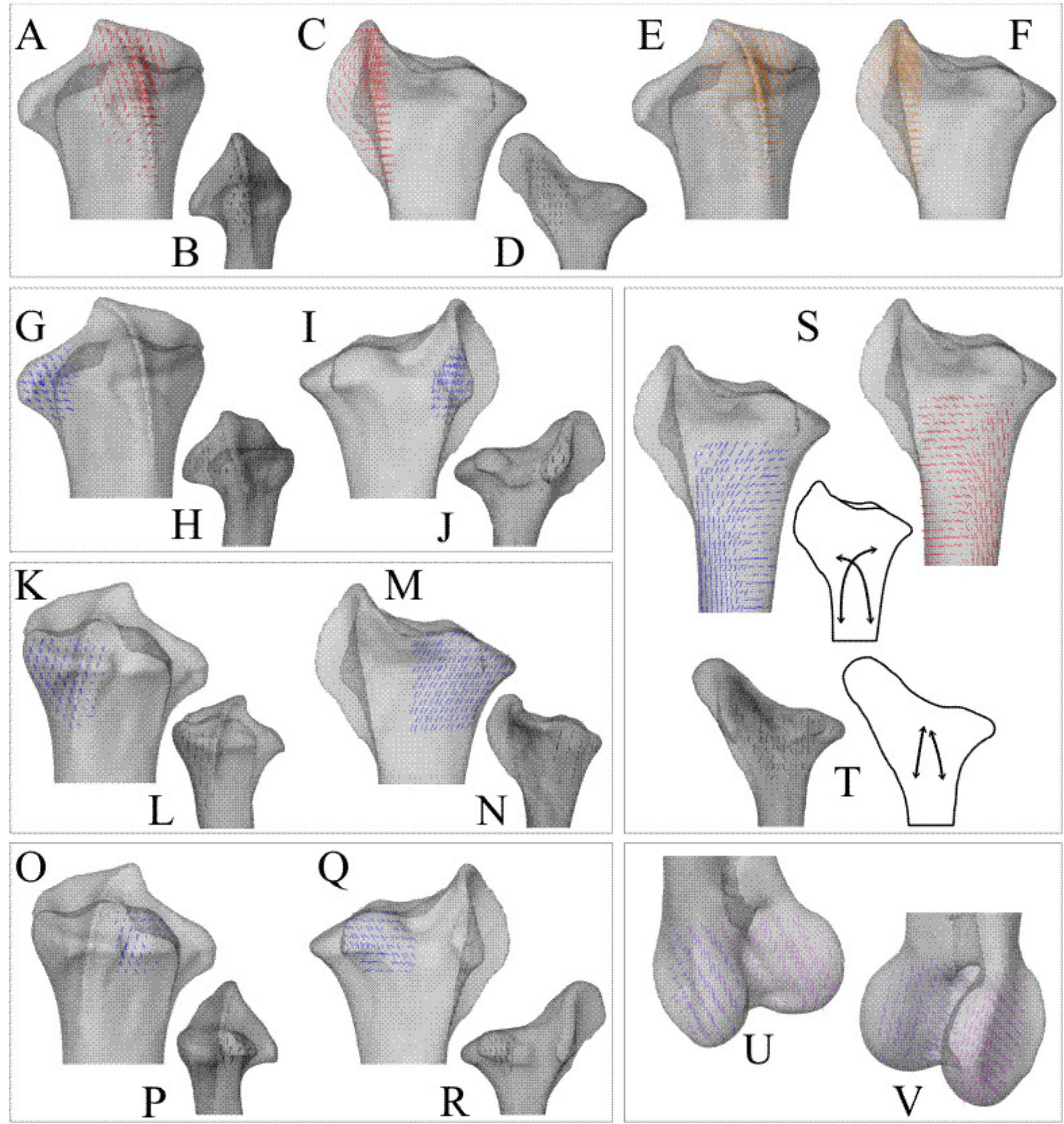


\section{Figure 7}

Principal stress trajectories for the fibula in the solution posture compared to cancellous bone fabric.

(A) Vector field of $\boldsymbol{\sigma}_{1}$ in the medial side of the fibular head plotted on a translucent rendering of the bone, in medial view (reversed). (B) Vector field of $\boldsymbol{\sigma}_{3}$ in lateral side of the fibular head, in lateral view. (C, D) Exemplar fabric vector fields $\left(\mathbf{u}_{1}\right)$ for birds, in lateral view (cf. Figs 40G-K of Part I); not to scale. 

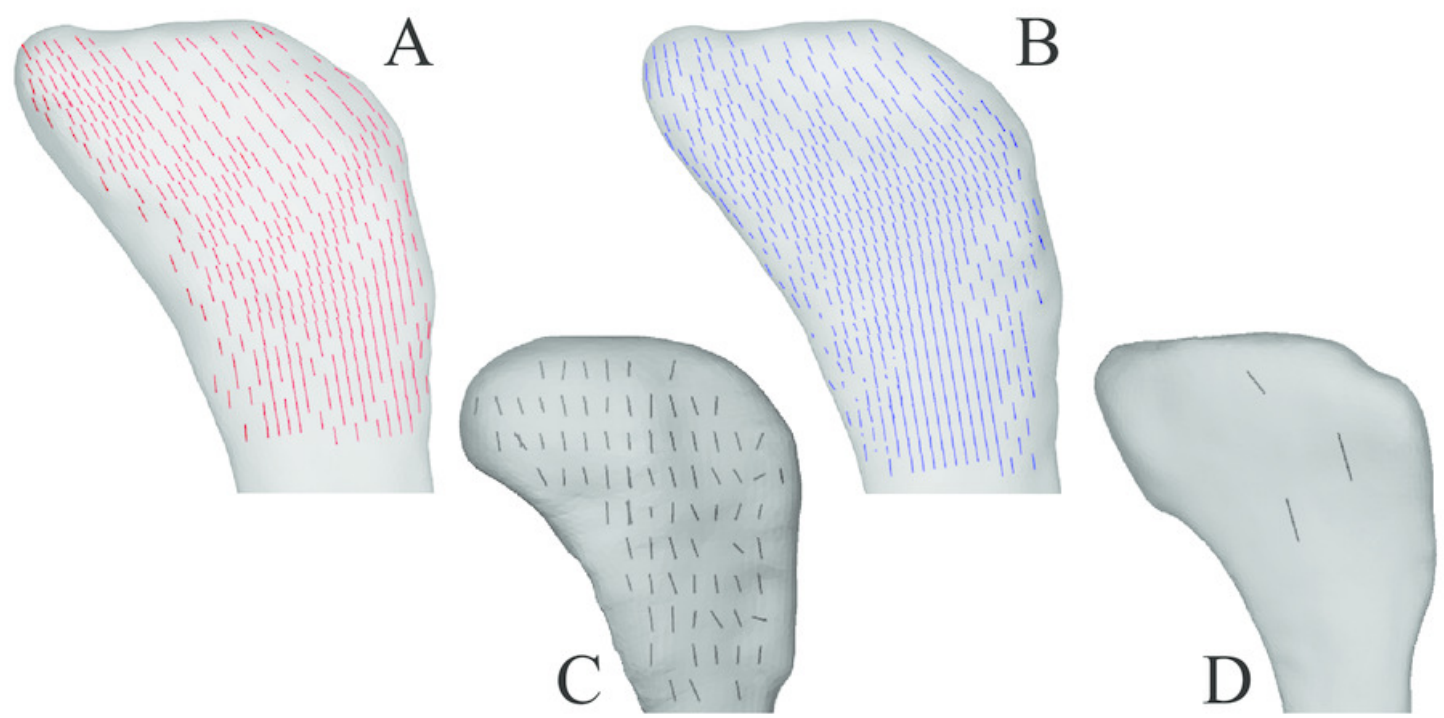


\section{Figure 8}

Additional aspects of the solution posture.

(A) The trajectories of the principal stresses $\boldsymbol{\sigma}_{1}$ (red) and $\boldsymbol{\sigma}_{3}$ (blue) at the femoral midshaft, in anterior view. (B) Oblique principal stresses in the femoral mid-shaft indicate of strong torsional loading (orange arrows), with a positive sense. (C) The trajectories of $\boldsymbol{\sigma}_{1}$ and $\boldsymbol{\sigma}_{3}$ at the tibial mid-shaft, in anterior view; note the almost complete lack of obliquity with respect to the long-axis of the bone. (D) Activations for each muscle actuator in the musculoskeletal simulation. In the original simulation all musculotendon actuators were assigned a maximum force of 2 body weights, whilst in the sensitivity test the assigned maximum force was specific to the size and architecture of each muscle. (E) Flexionextension muscle moment arms for the hip, knee and ankle joints; positive values indicate extension, negative values indicate flexion. For keys to abbreviations in $D$ and $E$, see Table 1 . 


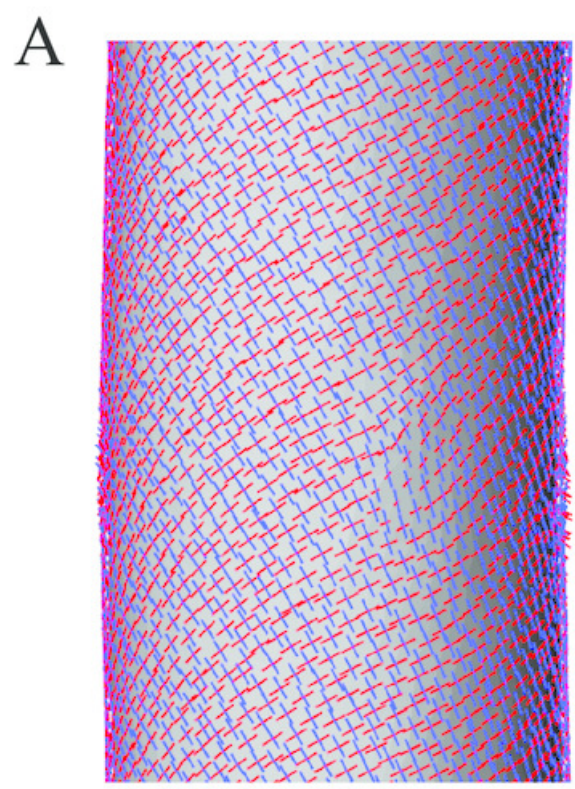

B

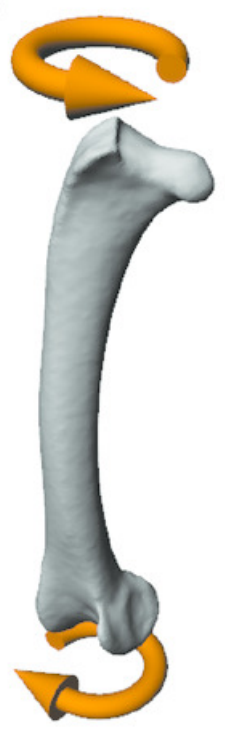

C

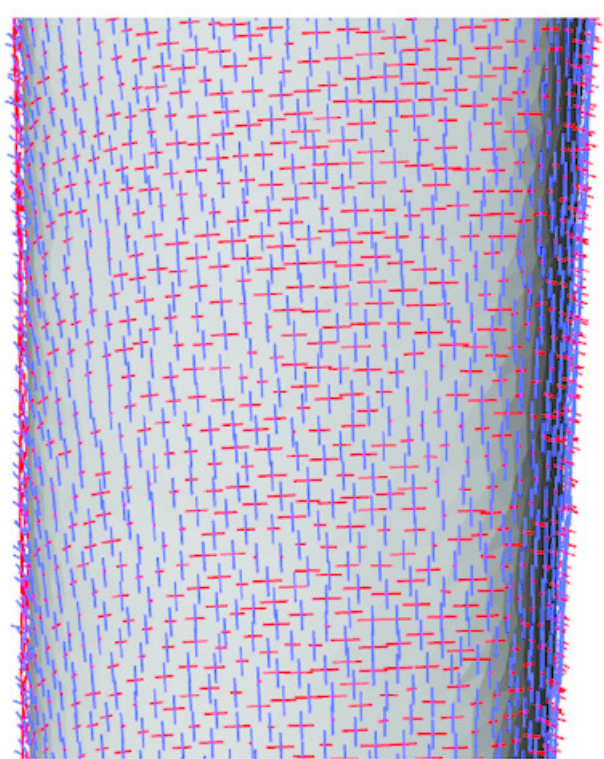

\section{D}
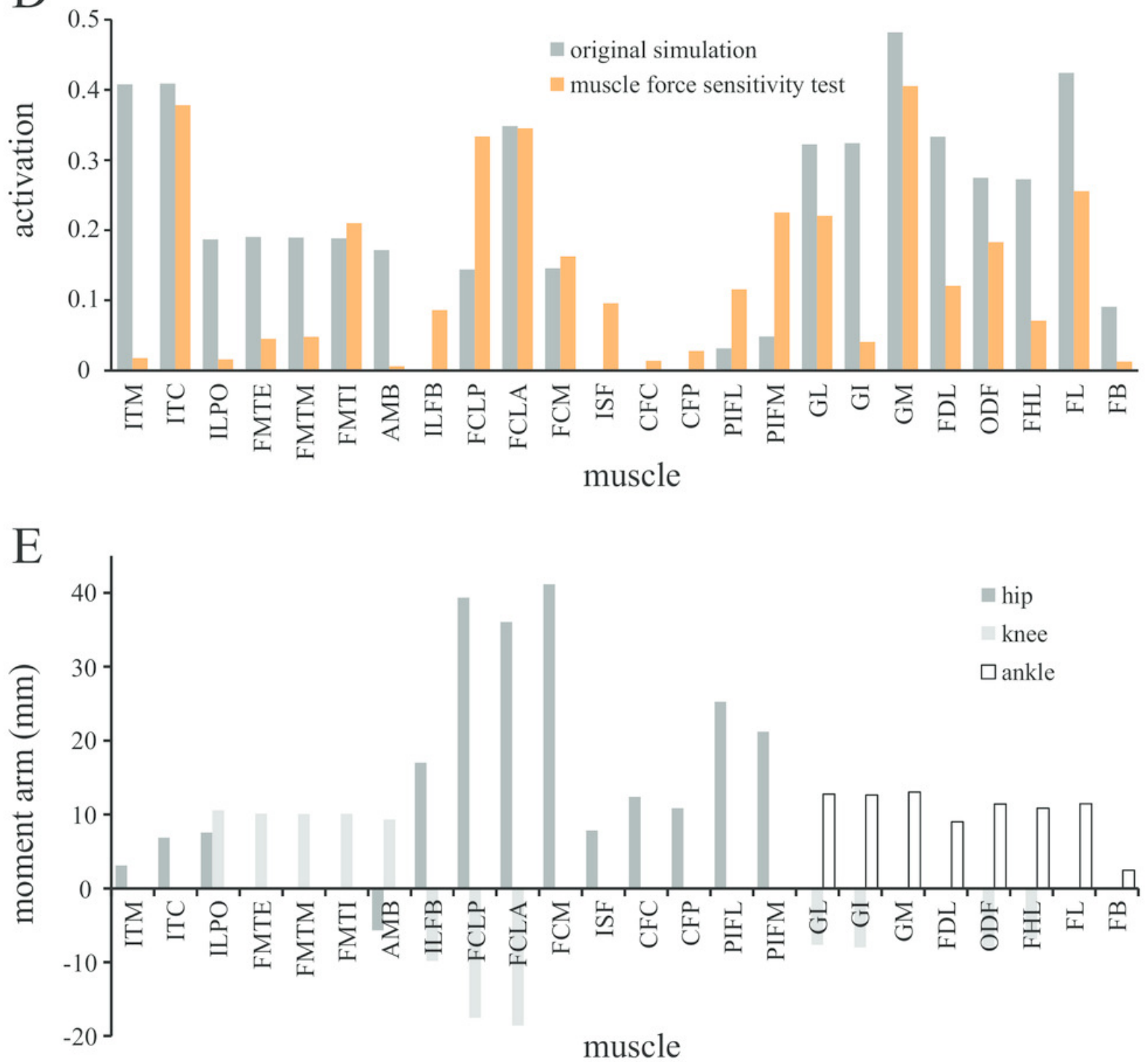